\title{
The chemical abundance analysis of normal early A- and late B-type stars ${ }^{\star} \star \star$
}

\author{
L. Fossati ${ }^{1}$, T. Ryabchikova ${ }^{1,2}$, S. Bagnulo ${ }^{3}$, E. Alecian ${ }^{4,5}$, J. Grunhut ${ }^{5}$, O. Kochukhov ${ }^{6}$, and G. Wade ${ }^{5}$ \\ 1 Institut für Astronomie, Universität Wien, Türkenschanstrasse 17, 1180 Wien, Austria \\ e-mail: [fossati;ryabchik]@astro.univie.ac.at \\ 2 Institute of Astronomy, Russian Academy of Sciences, Pyatnitskaya 48, 119017 Moscow, Russia \\ e-mail: ryabchik@inasan.ru \\ 3 Armagh Observatory, College Hill, Armagh BT61 9DG, Northern Ireland, UK \\ e-mail: sba@arm.ac.uk \\ 4 Observatoire de Paris-Meudon, LESIA, UMR 8111 du CNRS, 92195 Meudon Cedex, France \\ e-mail: evelyne.alecian@obspm.fr \\ 5 Physics Dept., Royal Military College of Canada, PO Box 17000, Station Forces, K7K 4B4, Kingston, Canada \\ e-mail: [Jason. Grunhut; Gregg]. Wade@rmc.ca \\ ${ }^{6}$ Department of Physics and Astronomy, Uppsala University, 75120 Uppsala, Sweden \\ e-mail: 0leg.Kochukhov@fysast.uu.se
}

Received 21 December 2008 / Accepted 19 May 2009

\section{ABSTRACT}

Context. Modern spectroscopy of early-type stars often aims at studying complex physical phenomena such as stellar pulsation, the peculiarity of the composition of the photosphere, chemical stratification, the presence of a magnetic field, and its interplay with the stellar atmosphere and the circumstellar environment. Comparatively less attention is paid to identifying and studying the "normal" A- and B-type stars and testing how the basic atomic parameters and standard spectral analysis allow one to fit the observations. By contrast, this kind of study is paramount for eventually allowing one to correctly quantify the impact of the various physical processes that occur inside the atmospheres of A- and B-type stars.

Aims. We wish to establish whether the chemical composition of the solar photosphere can be regarded as a reference for early Aand late B-type stars.

Methods. We have obtained optical high-resolution, high signal-to-noise ratio spectra of three slowly rotating early-type stars (HD 145788, $21 \mathrm{Peg}$ and $\pi$ Cet) that show no obvious sign of chemical peculiarity, and performed a very accurate LTE abundance analysis of up to 38 ions of 26 elements (for $21 \mathrm{Peg}$ ), using a vast amount of spectral lines visible in the spectral region covered by our spectra.

Results. We provide an exhaustive description of the abundance characteristics of the three analysed stars with a critical review of the line parameters used to derive the abundances. We compiled a table of atomic data for more than 1100 measured lines that may be used in the future as a reference. The abundances we obtained for $\mathrm{He}, \mathrm{C}, \mathrm{Al}, \mathrm{S}, \mathrm{V}, \mathrm{Cr}, \mathrm{Mn}, \mathrm{Fe}, \mathrm{Ni}, \mathrm{Sr}, \mathrm{Y}$, and $\mathrm{Zr}$ are compatible with the solar ones derived with recent 3D radiative-hydrodynamical simulations of the solar photosphere. The abundances of the remaining studied elements show some degree of discrepancy compared to the solar photosphere. Those of $\mathrm{N}, \mathrm{Na}, \mathrm{Mg}, \mathrm{Si}, \mathrm{Ca}, \mathrm{Ti}$, and $\mathrm{Nd}$ may well be ascribed to non-LTE effects; for $\mathrm{P}, \mathrm{Cl}$, Sc and Co, non-LTE effects are totally unknown; $\mathrm{O}, \mathrm{Ne}, \mathrm{Ar}$, and Ba show discrepancies that cannot be ascribed to non-LTE effects. The discrepancies obtained for O (in two stars) and Ne agree with very recent non-LTE abundance analysis of early B-type stars in the solar neighbourhood.

Key words. stars: abundances - stars: individual: HD 145788, 21 Peg, $\pi$ Cet

\section{Introduction}

In the last decade there has been dramatic improvement in the tools for the analysis of optical stellar spectra, both from the observational and theoretical perspective. New high-resolution echelle instruments have come online, which cover much broader spectral ranges than older single-order spectrographs. Data quality has also substantially improved in terms of signalto-noise ratio (SNR), because of substantially greater instrument efficiency, and the use of large-size telescopes. Thanks to the

\footnotetext{
* Figures 8-12 and Tables 6-8 are only available in electronic form at http://www . aanda. org

$\star \star$ Tables 9 is only available in electronic form at the CDS via anonymous ftp to cdsarc.u-strasbg. fr (130.79.128.5) or via http://cdsweb.u-strasbg.fr/cgi-bin/qcat?J/A+A/503/945
}

vibrant observational activities of the past few years, and thanks to efficient and user-friendly data archive facilities, a huge highquality spectroscopic database is now available to the public.

With the development of powerful and cheap computers, it has become practical to exploit these new data by performing spectral analysis using large spectral windows rather than selected spectral lines, at a level of realism heretofore impossible. The high accuracy of observations and modelling techniques now allows, for example, stretching the realm of abundance analysis to faster rotators than was possible in the past, but also provides the possibility of learning more about the structure of the stellar atmospheres and the ongoing physical processes, especially when spectral synthesis fails to reproduce the observations. For instance, observed discrepancies between observed and synthetic spectra have allowed us to discover that the 
signature of chemical stratification is ubiquitous in the spectra of some chemically peculiar stars (Bagnulo et al. 2001; Wade et al. 2001; Ryabchikova et al. 2003) and to perform accurate modelling of this stratification in the atmospheres of Ap stars (for instance, Ryabchikova et al. 2005; Kochukhov et al. 2006).

However, our inability to reproduce observations frequently stems for a very simple cause: that atomic data for individual spectral lines are incorrect. For solar type stars it is possible to construct a "reference" list of reliable spectral lines with reliable atomic data through the comparison of synthetic spectra with the solar observed spectrum, since the solar abundances are accurately known. In many cases, however, the solar spectrum cannot provide the required information, because the temperature of the target stars is significantly different from that of the sun. This problem can be overcome by adopting an analogous reference at a temperature reasonably close to the target temperature. The method involves a selection of a set of suitable reference stars for which very high quality spectra are available. Then an accurate determination of the stellar photospheric parameters and an accurate abundance analysis are performed with the largest possible number of spectral lines and the best possible atomic data. Finally, those spectral lines exhibiting the largest discrepancies from the model fit are identified, and their atomic data revised by assuming that the average abundance (inferred from the complete sample of spectral lines of that element) is the correct one. In this process it is important to take effects into account that can potentially play a significant role in all stellar atmospheres, such as variations in the model structure from non-solar abundances and non-LTE effects.

In this paper we address the problem of establishing references for effective temperatures around 10000-13000 K. This is in some respect the easiest temperature range to study, as well as one of the most interesting. This temperature is close to ideal because the spectra of stars in this interval are generally unaffected by severe blending. It is also relevant because stars in this temperature range display spectroscopic peculiarities (chemical abundance peculiarities, stratification, Zeeman effect, etc.) that reflect physical conditions and processes of interest for detailed investigation. A crucial prerequisite for studying and characterising these phenomena is the capacity to model the underlying stellar spectrum in detail, and this requires high quality atomic data.

The highest degree of accuracy in abundance analysis is reached for sharp-lined stars. Unfortunately, these objects are quite rare among A- and B-type stars, which are generally characterised by high rotational velocities. Furthermore, most of the slowly rotating stars in the chosen temperature region belong to various groups of magnetic and non-magnetic, chemically peculiar objects. As a matter of fact, many previous studies aimed at determining the chemical composition of "normal" early A- and late B-type stars were based on samples "polluted" by moderately chemically peculiar stars. For instance, the work by Hempel \& Holweger (2003) includes the sharp-lined HgMn star 53 Aur. Hill \& Landstreet (1993) searched for compositional differences among A-type stars, but four out of six programme stars are in fact classified as hot Am stars on the basis of the abundances of the heavy elements Sr-Y-Zr-Ba, which are considered as diffusion indicators (i.e., Sirius, $o$ Peg, and $\theta$ Leo, see Hempel \& Holweger 2003). The complexity of the problem of distinguishing between normal A and marginal Am stars is further stressed by Adelman \& Unsuree (2007). The aim of the present paper is to search for sharp-lined early A- or late B-type stars with a chemical composition as close as possible to the solar one. As a final outcome, one could assess whether the chemical composition of the solar photosphere may be considered at least in principle as a reference for the composition of the early A- and late B-type stars. If such a star is found, this will not imply that the solar composition is the most characteristic for the slowly rotating A- and B-type stars, but will be used as further evidence that any departure from the composition of the solar photosphere has to be explained in terms of diffusion or other physical mechanisms that are not active at the same efficiency level in the solar photosphere.

Our work is based on a very detailed and accurate study of a vast sample of spectral lines. As a by-product, we provide a list of more than 1100 spectral lines from which we have assessed the accuracy of the corresponding atomic data. Such a list may serve as a future reference for further abundance analysis studies of stars with a similar spectral type.

This paper is organised as follows. Section 2 describes the target selection, observations and data reduction, Sect. 3 presents methods and results for the choice of the best fundamental parameters that describe the atmospheres of the programme stars, Sect. 4 presents the methods and results of the abundance analysis of the programme stars. Our results are finally discussed in Sect. 5.

\section{Target selection, observations, and data reduction}

For our analysis we need a late A-type or early B-type star with a sharp-line spectrum (hence the star must have a small $v \sin i$ ) and one exhibiting the least possible complications due to phenomena such as non-homogeneous surface distribution of chemical elements, pulsation, or a magnetic field. Finding such a target is not a simple task, because most of the early-type stars are fast rotators. Slowly rotating A- and B-type stars generally show some type of chemical peculiarity often associated to the presence of abundance patches, a magnetic field (which broadens, or even splits spectral lines), and chemical stratification. Even Vega, which has been considered for a long time as the prototype of a "normal", slow rotating A-type star, is in fact currently classified as a $\lambda$ Boo star and discovered to actually be a fast rotating star seen pole-on, exhibiting, as such, distorted line profiles (Adelman \& Gulliver 1990; Yoon et al. 2008). Based on our knowledge, we reached the conclusion that the most suitable target for our project is the B9 star 21 Peg (HD 209459), which is known from previous studies as a "normal" single star with $v \sin i \sim 4 \mathrm{~km} \mathrm{~s}^{-1}$ (see, e.g. Sadakane 1981).

We felt it was necessary to consider additional targets of our spectral analysis, for two main reasons. Since we intend to provide an accurate reference for the typical abundances of the chemical elements in A- and B-type stars (and compare these values with the solar ones), we need to cross-check with further examples whether the results obtained for $21 \mathrm{Peg}$ are similar to those of other "normal", slow rotating A-type stars. Second, to check the accuracy of the astrophysical measurements of the $\log g f$ values, which is a natural complement of the present work. Both these goals are best achieved with the use of abundance values that have been obtained with a homogeneous method, rather than from a mixed collection of data from the literature. Therefore, we have also analysed another two stars of similar temperature as 21 Peg, i.e., HD 145788 (HR 6041) and $\pi$ Cet (HR 811). Both stars fulfill our requirements, although are slightly less ideal than 21 Peg. HD 145788 , suggested to us by Prof. Fekel, is a slowly rotating single star with $v \sin i \sim 8 \mathrm{~km} \mathrm{~s}^{-1}$ (Fekel 2003). $\pi$ Cet, a SB1 with 
$v \sin i \sim 20 \mathrm{~km} \mathrm{~s}^{-1}$, shows an infrared excess, and is a suspected Herbig Ae/Be star (Malfait et al. 1998). Since its spectrum is not visibly contaminated by the companion, it still serves our purpose. $\pi$ Cet was also already used as a normal comparison star in the abundance study of chemically peculiar stars by Smith \& Dworetsky (1993).

The star 21 Peg was observed five times during two observing nights in August 2007, with the FIES instrument of the North Optical Telescope (NOT). FIES is a cross-dispersed highresolution échelle spectrograph that offers a maximum spectral resolution of $R=65000$, covering the entire spectral range 3700-7400 A.

Data were reduced using a pipeline developed by Lyashko, which is based on the one described by Tsymbal et al. (2003). All bias and flat-field images were median-averaged before calibration, and the scattered light was subtracted by using a 2D background approximation. For cleaning cosmic ray hits, an algorithm that compares the direct and reversed observed spectral profiles was adopted. To determine the boundaries of echelle orders, the code uses a special template for each order position in each row across the dispersion axis. The shift of the row spectra relative to the template was derived by a cross-correlation technique. Wavelength calibration of each image was based on a single ThAr exposure, recorded immediately after the respective stellar time series, and calibrated by a 2D approximation of the dispersion surface. An internal accuracy of $\sim 100 \mathrm{~m} \mathrm{~s}^{-1}$ was achieved by using several hundred ThAr lines in every echelle order.

Each reduced spectrum has a SNR per pixel of about 300 at $5000 \AA$. All five spectra are fully consistent among themselves, which confirms that the star is not variable. This allowed us to combine all data in a unique spectrum with a final SNR of about 700 .

Because of the very low $v \sin i$ of $21 \mathrm{Peg}$, we made use of a very high-resolution spectrum $(R=120000)$ obtained with the Gecko instrument (now decommissioned) of the CanadaFrance-Hawaii Telescope (CFHT, Landstreet 1998) to measure this parameter. The spectrum covers the ranges $4612-4640 \AA$ and 5160-5192 $\AA$, which are too short to perform a full spectral analysis, but sufficient to measure $v \sin i$ with high accuracy. According to Hubrig et al. (2006) the mean longitudinal magnetic field of $21 \mathrm{Peg}$ is $-144 \pm 60 \mathrm{G}$, which excludes the possibility that the star has a structured magnetic field.

The spectrum of HD 145788 was obtained with the crossdispersed échelle spectrograph HARPS instrument attached at the 3.6-m ESO La Silla telescope, with an exposure time of $120 \mathrm{~s}$, and reduced with the online pipeline ${ }^{1}$. The reduced spectrum has a resolution of 115000 , and a SNR per pixel of about 200 at $5000 \AA$. The spectral range is $3780-6910 \AA$ with a gap between $5300 \AA$ and $5330 \AA$, because one echelle order is lost in the gap between the two chips of the CCD mosaic detector.

The star $\pi$ Cet was observed with the ESPaDOnS instrument of the CHFT February, 20 and 21 2005. ESPaDOnS consists of a table-top, cross-dispersed echelle spectrograph fed via a double optical fiber directly from a Cassegrain-mounted polarisation analysis module. Both Stokes $I$ and $V$ spectra were obtained throughout the spectral range 3700 to $10400 \AA$ at a resolution of about 65 000. The spectra were reduced using the Libre-ESpRIT reduction package Donati et al. (1997, and in prep.). The two spectra (each obtained from the combination of four $120 \mathrm{~s}$

\footnotetext{
${ }^{1}$ http://www.1s.eso.org/lasilla/sciops/3p6/harps/ software.html\#pipe
}

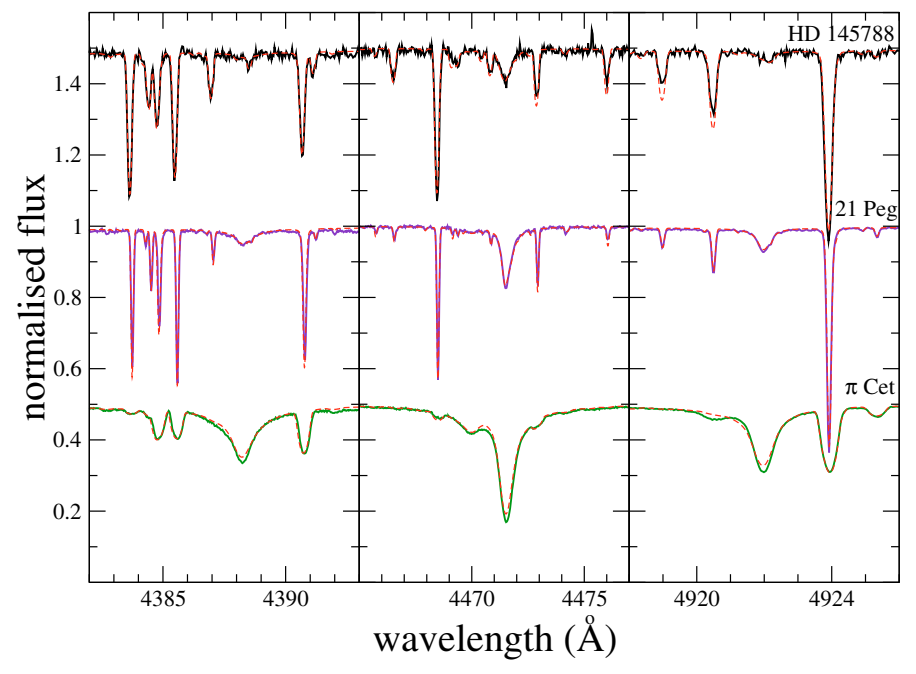

Fig. 1. Samples of the spectra of HD 145788, 21 Peg and $\pi$ Cet around three He I lines: $4387 \AA, 4471 \AA$ and $4921 \AA$ in comparison with our final synthetic profiles (dashed lines) calculated for each line. The spectra of HD 145788 and $\pi$ Cet are shifted upwards and downwards of 0.5, respectively.

sub-exposures) were combined into a final spectrum that has an SNR per pixel of about 1200 at $5000 \AA$.

The observation of $\pi$ Cet enters in the context of a large spectropolarimetric survey of Herbig Ae/Be stars. Least-squares deconvolution (LSD, Donati et al. 1997) was applied to the spectra of $\pi$ Cet assuming a solar abundance line mask corresponding to an effective temperature of $13000 \mathrm{~K}$. The resulting LSD profiles show a clean, relatively sharp mean Stokes $I$ profile, corresponding to $v \sin i=20 \pm 1 \mathrm{~km} \mathrm{~s}^{-1}$, and no detection of any Stokes $V$ signature indicative of a photospheric magnetic field. Integration of Stokes $V$ across the line using Eq. (1) of Wade et al. (2000) yields longitudinal magnetic fields consistent with zero field and with formal $1 \sigma$ uncertainties of about $10 \mathrm{G}$. The high-resolution spectropolarimetric measurements therefore provide no evidence of magnetic fields in the photospheric layers of the star.

All the spectra of the three stars were normalised by fitting a spline to carefully selected continuum points. For each object, radial velocities $v_{\mathrm{r}}$ were determined by computing the median of the results obtained by fitting synthetic line profiles of several individual carefully selected lines into the observed spectrum. The $v_{\mathrm{r}}$ values are listed in Table 1, and their uncertainty is of the order of $0.5 \mathrm{~km} \mathrm{~s}^{-1}$. The spectra were then shifted to the wavelength rest frame. Selected spectral windows containing the observed blue He I lines, together with the synthetic profiles, are displayed in Fig. 1.

\section{Fundamental parameters}

Fundamental parameters for the atmospheric models were obtained using photometric indicators as a first guess. For their refined estimate, we performed a spectroscopic analysis of hydrogen lines and metal lines, and as a final step, compared the observed and computed energy distributions. The spectroscopic tuning of the fundamental parameters is needed since different photometries and calibrations would give different parameters and uncertainties. The spectroscopic analysis will provide a set of parameters that fit all the parameter indicators consistently, with less uncertainties. Model atmospheres were calculated with LLMODELS, an LTE code that uses direct sampling of the line 
Table 1. Adopted atmospheric parameters for the analysed stars.

\begin{tabular}{cccccccc}
\hline \hline $\begin{array}{c}\text { Star } \\
\text { Name }\end{array}$ & $\begin{array}{c}T_{\text {eff }} \\
{[\mathrm{K}]}\end{array}$ & $\begin{array}{c}\log g \\
{[\mathrm{cgs}]}\end{array}$ & $\begin{array}{c}v_{\text {mic }} \\
{\left[\mathrm{km} \mathrm{s}^{-1}\right]}\end{array}$ & $\begin{array}{c}\sigma_{v_{\text {mic }}} \\
{\left[\mathrm{km} \mathrm{s}^{-1}\right]}\end{array}$ & $\begin{array}{c}v \sin i \\
{\left[\mathrm{~km} \mathrm{~s}^{-1}\right]}\end{array}$ & $\begin{array}{c}\sigma_{v \sin i} \\
{\left[\mathrm{~km} \mathrm{~s}^{-1}\right]}\end{array}$ & $\begin{array}{c}v_{\mathrm{r}} \\
{\left[\mathrm{km} \mathrm{s}^{-1}\right]}\end{array}$ \\
\hline 21 Peg & 10400 & 3.55 & 0.5 & 0.4 & 3.76 & 0.35 & 0.5 \\
HD 145788 & 9750 & 3.70 & 1.3 & 0.2 & 10.0 & 0.5 & -13.9 \\
$\pi$ Cet & 12800 & 3.75 & 1.0 & 0.5 & 20.2 & 0.9 & 12.5 \\
\hline
\end{tabular}

The uncertainties on $T_{\text {eff }}, \log g$, and $v_{\mathrm{r}}$ are $200 \mathrm{~K}, 0.1 \mathrm{dex}$, and $0.5 \mathrm{~km} \mathrm{~s}^{-1}$, respectively.

opacities (Shulyak et al. 2004) and allows computing models with an individualised abundance pattern. Atomic parameters of spectral lines used for model atmosphere calculations were extracted from the VALD database (Piskunov et al. 1995; Kupka et al. 1999; Ryabchikova et al. 1999).

Before applying the spectroscopic method, we estimated the star's $v \sin i$. For $21 \mathrm{Peg}$, a $v \sin i$ value of $3.76 \pm 0.35 \mathrm{~km} \mathrm{~s}^{-1}$ was derived from the fit with a synthetic spectrum to 21 carefully selected lines observed with the Gecko instrument. This value agrees very well with the $3.9 \mathrm{~km} \mathrm{~s}^{-1}$ value derived by Landstreet (1998). Achieving such high precision was possible thanks to the high quality of the spectrum and the low $v \sin i$. The $v \sin i$ values for HD 145788 and $\pi$ Cet, $10.0 \pm 0.5 \mathrm{~km} \mathrm{~s}^{-1}$ and $20.2 \pm$ $0.9 \mathrm{~km} \mathrm{~s}^{-1}$, respectively, were derived from fitting about 20 wellselected lines along the whole available spectral region.

In the next sections, we describe the determination of the atmospheric parameters: $T_{\text {eff }}$, effective gravity $(\log g)$, and microturbulent velocity $\left(v_{\text {mic }}\right)$, and their uncertainties. The fundamental parameters finally adopted for $21 \mathrm{Peg}$, HD 145788, and $\pi$ Cet are given in Table 1 .

\subsection{Photometric indicators}

Since none of the three programme stars, HD 145788, $21 \mathrm{Peg}$ or $\pi$ Cet are known to be photometrically variable or peculiar, we can use temperature and gravity calibrations of different photometric indices for normal stars to get a preliminary estimate of the atmospheric parameters. The effective temperature $\left(T_{\text {eff }}\right)$ and gravity $(\log g)$ were derived from Strömgren photometry (Hauck \& Mermilliod 1998) with calibrations by Moon \& Dworetsky (1985) and by Napiwotzki et al. (1993), and from Geneva photometry (Rufener 1988) with the calibration by North \& Nicolet (1990). The mean parameters from the three calibrations that were used as starting models are the following ones: $T_{\text {eff }}=9675 \pm 75 \mathrm{~K}, \log g=3.72 \pm 0.03$ for HD 145788; $T_{\text {eff }}=10255 \pm 115 \mathrm{~K}, \log g=3.51$ for $21 \mathrm{Peg} ; T_{\text {eff }}=13200 \pm$ $65 \mathrm{~K} \log g=3.77 \pm 0.15$ for $\pi$ Cet. No error bar is given for the $\log g$ of 21 Peg since all three calibrations give the same value.

\subsection{Spectroscopic indicators}

\subsubsection{Hydrogen lines}

For a fully consistent abundance analysis, the photometric parameters have to be checked and eventually tuned according to spectroscopic indicators, such as hydrogen line profiles. In the temperature range where HD 145788, $21 \mathrm{Peg}$, and $\pi$ Cet lie, the hydrogen line wings are less sensitive to $T_{\text {eff }}$ than to $\log g$ variations, but temperature effects can still be visible in the part of the wings close to the line core. For this reason hydrogen lines are very important not only for our analysis, but in general for every consistent parameter determination. To spectroscopically derive the fundamental parameters from hydrogen lines, we fitted synthetic line profiles, calculated with SynTH3 (Kochukhov 2007), to the observed profiles. Synth3 incorporates the code by Barklem et al. $(2000)^{2}$ that takes into account not only self-broadening but also Stark broadening (see their Sect. 3). For the latter, the default mode of SyNTH3, adopted in this work, uses an improved and extended HLINOP routine (Kurucz 1993).

Figure 2 shows the comparison between the observed $\mathrm{H} \beta$ line profile for $21 \mathrm{Peg}$ and the synthetic profiles calculated with the adopted stellar parameters. In Fig. 2 we also added the synthetic line profiles calculated with $1 \sigma$ error bars on $T_{\text {eff }}( \pm 200 \mathrm{~K}$; upper profile) and $\log g( \pm 0.1 \mathrm{dex}$; lower profile). The same profiles with the same uncertainties, but for $\mathrm{H} \gamma, \mathrm{H} \beta$, and $\mathrm{H} \alpha$ (from left to right) for the three programme stars, are shown in Figs. 9, 8, and 10 (online material).

The three hydrogen lines $(\mathrm{H} \gamma, \mathrm{H} \beta$, and $\mathrm{H} \alpha)$ used to spectroscopically improve the fundamental parameters for HD 145788 gave slightly different results both for $T_{\text {eff }}$ and for $\log g$. As final values, we took their mean $\left(T_{\text {eff }}=9750 \mathrm{~K} ; \log g=3.7\right)$. This discrepancy is visible in Fig. 9 (online material), but it lies within the errors given for $T_{\text {eff }}$ and $\log g$. The spectrum of HD 145788 also allowed a good normalisation of the region bluer than $\mathrm{H} \gamma$. We synthesised this region to check the quality of our final fundamental parameters finding a very good fit for the three hydrogen lines $\mathrm{H} \delta, \mathrm{H} \epsilon$, and $\mathrm{H}_{8}$.

For $21 \mathrm{Peg}$ we obtained the same temperature estimates from $\mathrm{H} \alpha$ and $\mathrm{H} \beta\left(T_{\text {eff }}=10400 \mathrm{~K}\right)$ and by $200 \mathrm{~K}$ less from the fitting of $\mathrm{H} \gamma$. We adopted a final $T_{\text {eff }}$ of $10400 \mathrm{~K}$. To fit all three hydrogen lines, we need slightly different values of $\log g: 3.47$ for $\mathrm{H} \gamma$, 3.54 for $\mathrm{H} \beta$ and 3.57 for $\mathrm{H} \alpha$. We finally adopted $\log g=3.55$ taking possible continuum normalisation problems into account, in particular, for $\mathrm{H} \gamma$.

The temperature determination for $\pi$ Cet was more difficult thanks to the weak effect that this parameter has on the hydrogen lines at about $13000 \mathrm{~K}$ and to the slightly peculiar shape of the $\mathrm{H} \alpha$ line. As explained in Sect. 5, $\pi$ Cet probably shows a small emission signature in the region around the core of $\mathrm{H} \alpha$ possibly because of a circumstellar disk. This region is the one where $T_{\text {eff }}$ effects are visible, making it almost impossible to obtain a good temperature determination from this line. $\mathrm{H} \gamma$ and $\mathrm{H} \beta$ gave best temperatures of $12700 \mathrm{~K}$ and $12900 \mathrm{~K}$, respectively, leading to a final adopted value of $12800 \mathrm{~K}$. Confirmation of this value was then given by the spectrophotometry (see Sect. 3.3). The results of LTE abundance analysis (Sect. 4) show that $\pi$ Cet has little He overabundance that leads to an overestimation of $\log g$ if the He abundance is not taken into account in the model atmosphere calculation (Auer et al. 1966). For this reason we derived the first set of fundamental parameters $\left(T_{\mathrm{eff}}=12800 \mathrm{~K} ; \log g=3.80\right)$ and then the He abundance $\left(\log \left(N_{\mathrm{He}} / N_{\mathrm{tot}}\right)=-0.97 \mathrm{dex}\right)$. As a next step we recalculated a set of model atmospheres with the derived He abundance and re-fit the hydrogen line profiles. The best-fit gave us the same temperature, but weak effective gravity $(\log g=3.75)$. As expected, the He overabundance is acting as pressure, requiring an adjustment of $\log g$ to be balanced. We

${ }^{2}$ http://www.astro.uu.se/ barklem/hlinop.html 


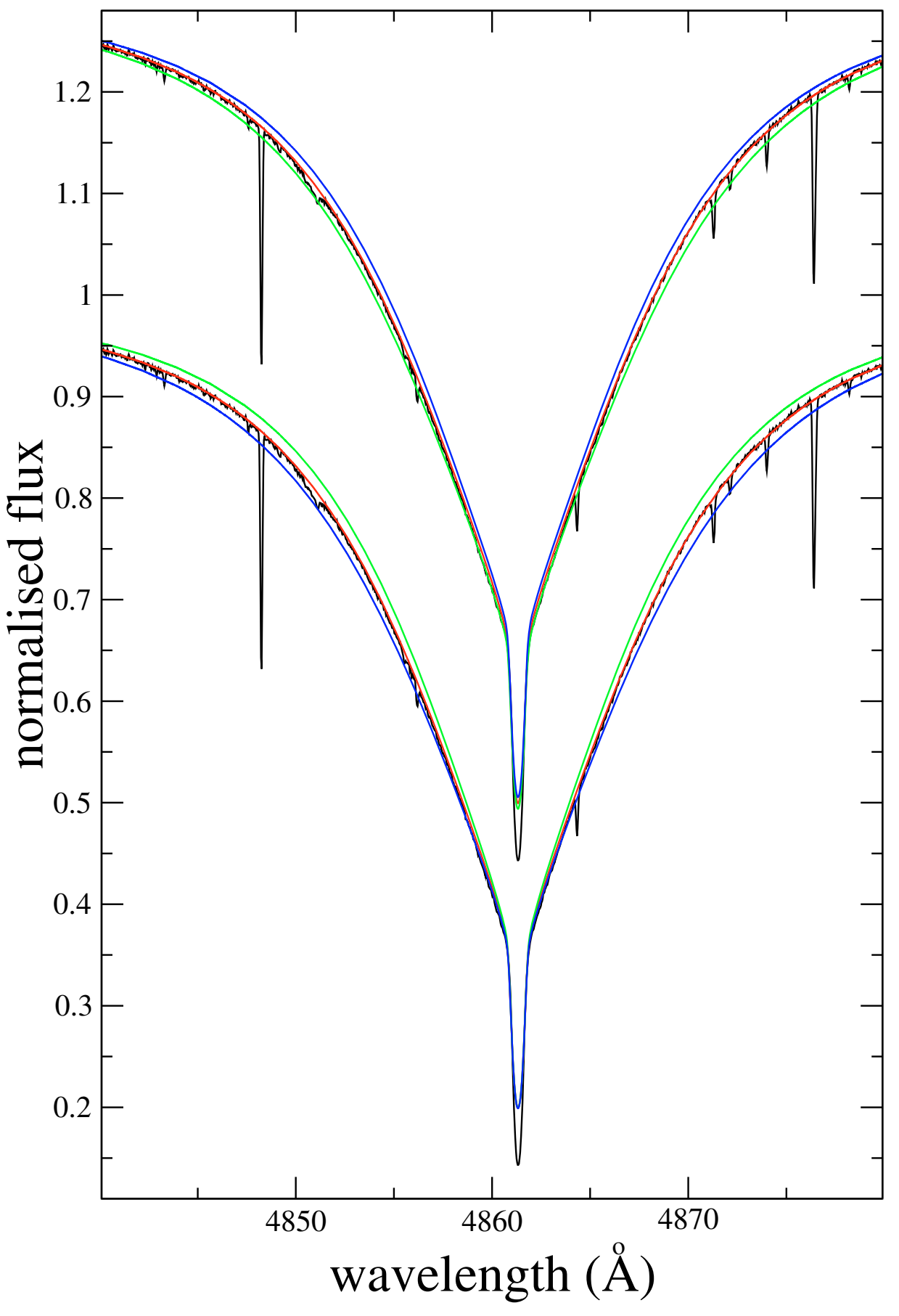

Fig. 2. Observed $\mathrm{H} \beta$ line profile for $21 \mathrm{Peg}$ (black solid line), compared to synthetic profiles (red, blue, and green lines). The red solid lines were obtained assuming the best $T_{\text {eff }}$ and $\log g$ values of Table 1 . The blue lines show the synthetic profile by increasing $T_{\text {eff }}$ by $200 \mathrm{~K}$ (top spectrum) or $\log g$ by 0.1 dex (bottom spectrum). The green lines show the synthetic profile by decreasing $T_{\text {eff }}$ by $200 \mathrm{~K}$ (top spectrum) or $\log g$ by $0.1 \mathrm{dex}$ (bottom spectrum). obtained the He abundance from the fitting of the blue He line wings. The blue He lines are, in general, considered as showing very little non-LTE effect (Leone \& Lanzafame 1998), and as we only used the line wings, this leads us to conclude that our results should not be affected by non-LTE effects and that the $\mathrm{He}$ is overabundant in $\pi$ Cet. The best fit to the blue He I lines of $\pi$ Cet is shown in Fig. 1.

The example of $\pi$ Cet is important because it clearly shows the effect of the single element abundance on the parameter determination, not only for chemically peculiar stars (for which this effect is well known and often, but not always, taken into account), but also for chemically "normal" stars.

The set of parameters that best fit the hydrogen line profiles could not be unique. For $21 \mathrm{Peg}$, we checked that using a combination of a lower temperature and lower gravity or else higher temperature and higher gravity increases the standard deviation of the fit of the $\mathrm{H} \beta$ line wings by $\sim 25 \%$. The result is that a different combination of $T_{\text {eff }}$ and $\log g$ could in principle provide a similar fit. For this reason the derived fundamental parameters should be checked with other indicators, such as the analysis of metallic lines (ionisation and excitation equilibrium) and the fitting of the spectral energy distribution. The latter is more important because ionisation and excitation equilibrium should be strictly used only with a full non-LTE treatment of the line formation.

\subsubsection{Metallic lines}

The metallic-line spectrum may also provide constraints on the atmospheric parameters. If no deviation from the local 

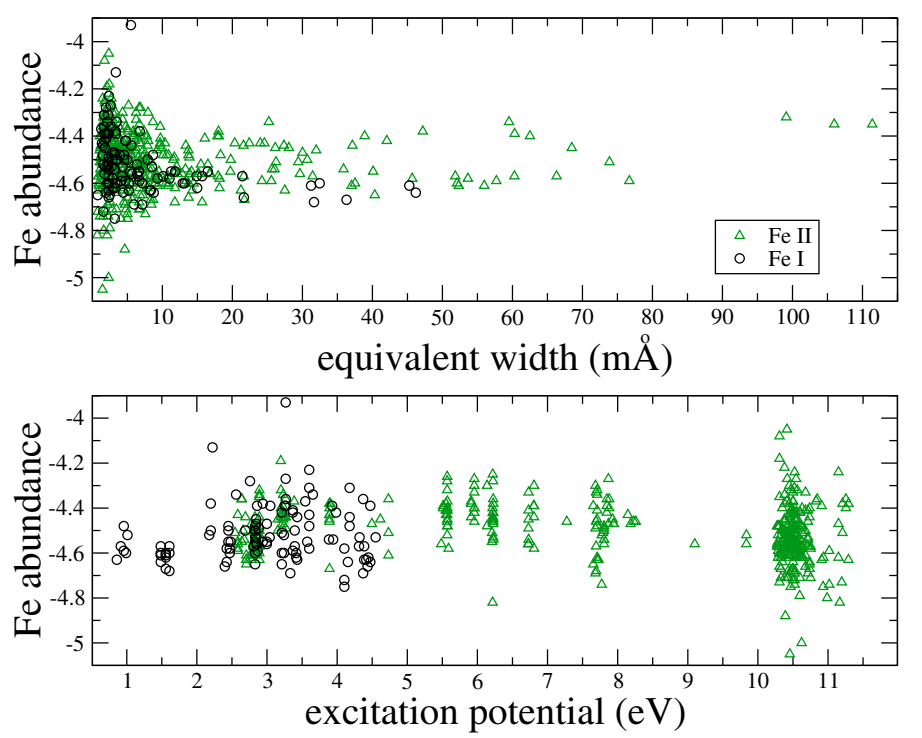

Fig. 3. Iron abundance vs. equivalent widths (upper panel) and excitation potential (lower panel) for $21 \mathrm{Peg}$. The open circles indicate Fe I, while the open triangles indicate Fe II.

thermodynamic equilibrium (LTE) is expected, the minimisation of the correlation between individual line abundances and excitation potential, for a certain element/ion, allows one to check the determined $T_{\text {eff }}$. Then the balance between different ionisation stages of the same element provides a check for log $g$. The microturbulent velocity, $v_{\text {mic }}$ is then determined by minimising the correlation between individual abundances and equivalent widths for a certain element. Determining the fundamental parameters performed in this way has to be done iteratively since, for example, a variation in $T_{\text {eff }}$ leads to a change in the best $\log g$ and $v_{\text {mic }}$.

Figure 3 shows the correlations of Fe I and Fe II abundances with the equivalent widths (upper panel) and with the excitation potential (lower panel) for $21 \mathrm{Peg}$. The correlation with the equivalent widths is shown for a $v_{\text {mic }}$ of $0.5 \mathrm{~km} \mathrm{~s}^{-1}$, which we infer to be the best value for $21 \mathrm{Peg}$, since the slope of the linear fit for Fe I is $-4.897 \times 10^{-3} \pm 1.560 \times 10^{-3} \mathrm{~mA}^{-1}$ and for Fe II is $2.146 \times 10^{-5} \pm 4.058 \times 10^{-4} \mathrm{~m}^{-1}$. Here we gave a preference to the result obtained from Fe II because of the higher number of measured Fe II lines in a wider range of equivalent widths. The same analysis was made for HD 145788 and for $\pi$ Cet. The error bar on $v_{\text {mic }}$ was calculated using the error bar of the slope (abundance vs. equivalent width) derived from a set of different $v_{\text {mic }}$. The uncertainties listed in Table 1 are given considering $2 \sigma$ on the error bar of the derived slopes. Considering a $1 \sigma$ error bar, the uncertainties on $v_{\text {mic }}$ are of $0.1 \mathrm{~km} \mathrm{~s}^{-1}$ for HD 145788 and $21 \mathrm{Peg}$ and of $0.2 \mathrm{~km} \mathrm{~s}^{-1}$ for $\pi$ Cet.

According to previous works, deviation from LTE of the Fe II lines is expected to be very small ( $~ 0.02$ dex Gigas 1986; Rentzsch-Holm 1996) for the analysed stars, such that the absence of any clear correlation between the line Fe II abundance and the excitation potential confirms the $T_{\text {eff }}$ derived from the hydrogen lines.

For the Fe I lines, deviations from LTE of about +0.3 dex are given by Gigas (1986) and Rentzsch-Holm (1996). However, both Gigas (1986) and Rentzsch-Holm (1996), as well as Hempel \& Holweger (2003), used the same model atom, which includes $79 \mathrm{Fe}$ I and $20 \mathrm{Fe}$ II energy levels. We note that the highest energy level in their model atom for Fe II has an excitation energy of about $6 \mathrm{eV}$, while the ionisation potential is $16.17 \mathrm{eV}$.
Such a model atom does not provide collisional coupling of Fe II to Fe III, which operates for the majority of iron atoms in line formation layers below $\log \tau_{5000}=-1$. Unfortunately, the existing NLTE calculations for Fe are not accurate enough to be applied now to our stars. Clearly, an extended energy-level model atom is needed for a reliable non-LTE analysis of Fe. The ionisation equilibrium for different elements/ions (or its violation) can be seen in Table 4 and is discussed in Sect. 4.

\subsection{Spectrophotometry}

For a complete self-consistent analysis of any star, one should reproduce the observed spectral energy distribution with the adopted parameters for a model atmosphere. In the optical spectral region, spectrophotometry was only available for $21 \mathrm{Peg}$ and $\pi$ Cet, while in the ultraviolet, IUE spectra were available for all three stars. For $\pi$ Cet ultraviolet spectrophotometry from the TD-1 satellite (Jamar et al. 1976) was also available, along with the flux calibrated spectra from STIS at HST (Gregg et al. 2004). The comparison between the observed flux distributions and the model fluxes calculated with the adopted atmospheric parameters for 21 Peg and $\pi$ Cet is shown in Fig. 4. For HD 145788 we estimated a reddening $E(B-V) \approx 0.2$ from the dust maps of Schlegel et al. (1998). The comparison of reddened model fluxes with the available IUE spectrum, Johnson UBV photometry (Nicolet 1978), Geneva photometry ${ }^{3}$ and 2MASS photometry (Cutri et al. 2003) is shown in Fig. 11 (online material). This plots supports the value of $E(B-V)=0.2$ and shows good agreement between all the observations and the model fluxes, confirming the obtained fundamental parameters, and also the importance of considering reddening in the analysis of relatively nearby stars such as HD 145788.

The optical spectrophotometry was taken from Adelman et al. (1989) and Breger (1976). All flux measurements were normalised to the flux value at $5000 \AA$ obtained from observed data for $\pi$ Cet and 21 Peg and from the model fluxes for HD 145788. Also for $21 \mathrm{Peg}$ and $\pi$ Cet, we extended the comparison to the near infrared region including 2MASS photometry (Cutri et al. 2003). Following Netopil et al. (2008), we obtained a reddening $E(B-V) \leq 0.01 \pm 0.01 \mathrm{mag}$ for both $\pi$ Cet and $21 \mathrm{Peg}$. A good agreement between the unreddened model fluxes and the observed spectrophotometry from UV to near infrared confirms negligible reddening for these two stars.

The agreement between the IUE observation and the model fluxes is very good for HD 145788, but the IUE data alone cannot be used as a check of $T_{\text {eff }}$, since a variation of about $1000-1500 \mathrm{~K}$ is needed to make any visible discrepancy. At the $T_{\text {eff }}$ of HD 145788 hydrogen lines were still quite sensitive to temperature variations, so we did not need accurate spectrophotometry for a reliable effective temperature estimate.

The model fluxes of $21 \mathrm{Peg}$ are in good agreement with the observations from the ultraviolet up to the near infrared. For 21 Peg and $\pi$ Cet, few data points in the red appear above the model fluxes. This often happens with the optical spectrophotometry as shown in Fig. 1 of Adelman et al. (2002). We do not have a definite explanation for this effect, although taking a very accurate measurement of the reddening into account could remove this inconsistency. As for the hydrogen lines, we checked the fit of the spectral energy distribution to model fluxes calculated with a combination of a lower temperature and lower

${ }^{3}$ http://obswww.unige.ch/gcpd/ph13.html 


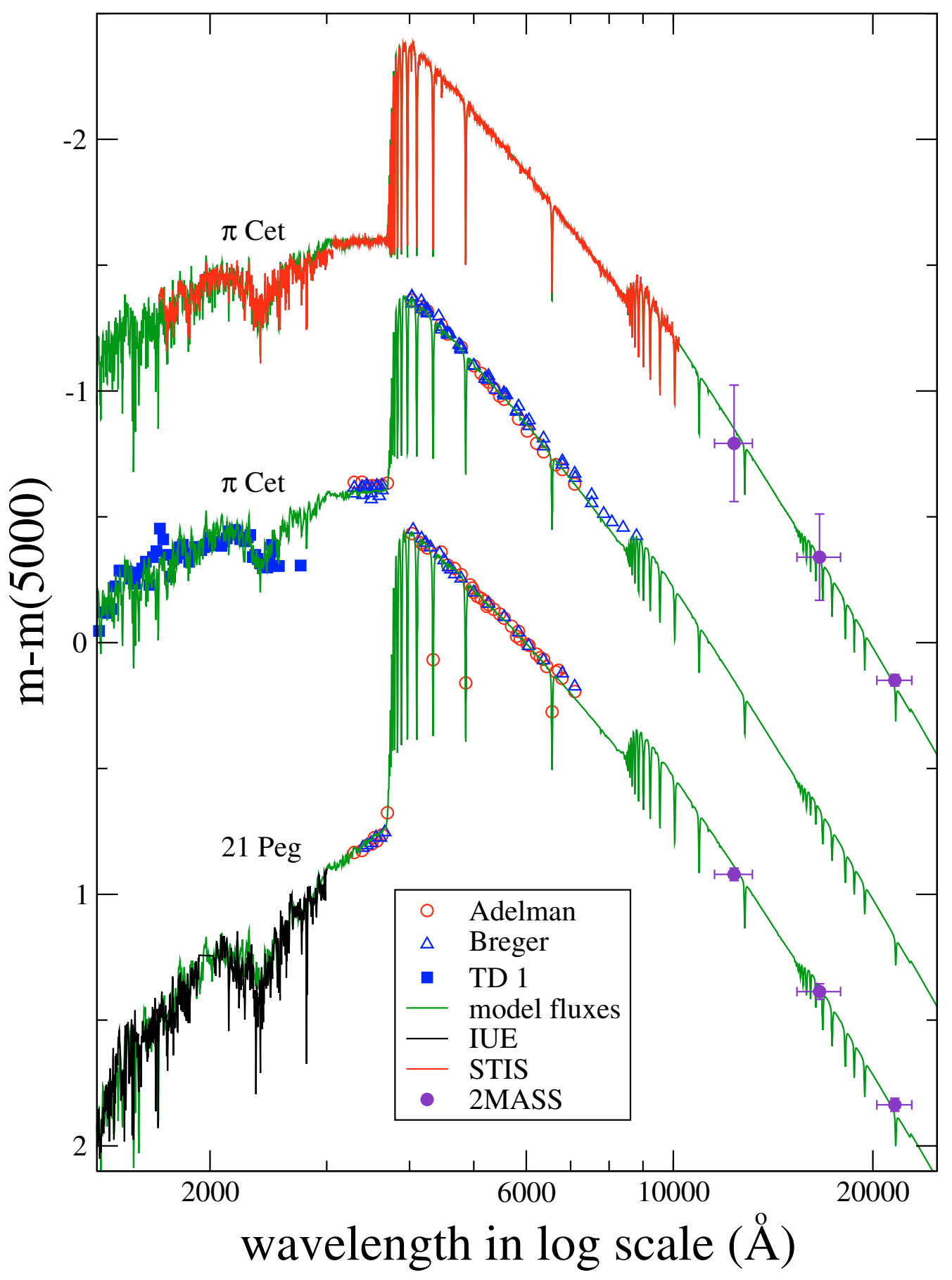

Fig. 4. Comparison between LLMODELS theoretical fluxes calculated with the fundamental parameters and abundances derived for $21 \mathrm{Peg}$ and $\pi$ Cet (green solid line) with spectrophotometries by Adelman et al. (1989) (open red circles) and Breger (1976) (open blue triangles), IUE calibrated fluxes (full black line), TD 1 observations (full blue squares) and STIS spectrum (full red line). The violet full dots represent the 2MASS photometry for which the given error bar in wavelength shows the wavelength range covered by each filter. The model fluxes for $21 \mathrm{Peg}$ and $\pi$ Cet, shown in the middle of the panel, were convolved to have the same spectral resolution of the IUE fluxes $(R \sim 900)$, while the upper of $\pi$ Cet has a spectral resolution 700 , approximately the same of STIS spectra. For a better visualisation of the whole spectral region the $x$-axis is on a logarithmic scale. gravity or a higher temperature and higher gravity. In both cases the fit clearly becomes worse mainly in the spectral region around the Balmer jump. This definitively excludes the possibility that a combination of fundamental parameters, which gives a worse but still reasonable fit to the hydrogen line profiles, should be considered.

For $\pi$ Cet we obtained several spectrophotometric observations from different sources. In particular, the data in the optical region around the Balmer jump were conclusive for $T_{\text {eff }}$ determination, as mentioned already in Sect. 3.2.1. The STIS spectrum shown in Fig. 4 is formed by three separated spectra (UV, visible, IR) with about the same spectral resolution. We noticed a small (but not negligible) vertical jump at the overlapping wavelengths between the three spectra. For this reason we decided to fit them separately. This decision was based on an adjustment performed at the level of the flux calibration (Maíz-Apellániz 2005, 2006).

The use of an energy distribution plays a crucial role in adjusting the atmospheric parameters because it is independent of the photometric calibrations (different for each author), of the hydrogen line fitting (reduction and normalisation dependent), and of the ionisation equilibria approach (dependent on several effects such as the adopted atomic line data and non-LTE effects).

The uncertainties on $T_{\text {eff }}$ and $\log g$ were derived from the hydrogen line fitting. This way of deriving the error bars on the parameters also includes the SNR of the observations. For all three stars, we derived an error bar on the $T_{\text {eff }}$ of $200 \mathrm{~K}$ and 0.1 dex in $\log g$, as also shown in Figs. 8-10 (online material). 
Table 2. Atmospheric parameters of 21 Peg derived from other authors.

\begin{tabular}{cccc}
\hline \hline Reference & $\begin{array}{c}T_{\text {eff }} \\
{[\mathrm{K}]}\end{array}$ & $\begin{array}{c}\log g \\
{[\mathrm{cgs}]}\end{array}$ & Method \\
\hline S81 & 10500 & 3.50 & SPh, JPh, Fe eq, H-fit, SED \\
B82 & 10500 & 3.50 & SPh, H-fit \\
A83 & 10700 & $(\ldots)$ & JPh \\
A83 & 10350 & $(\ldots)$ & SPh \\
A83 & 10375 & $(\ldots)$ & SED \\
M85 & 10300 & $(\ldots)$ & SED \\
S93 & 10450 & 3.50 & SPh, H-fit \\
L98 & 10200 & 3.50 & SPh \\
W00 & 10350 & 3.48 & SPh \\
H01 & 10450 & 3.60 & SPh \\
A02 & 10375 & 3.47 & SPh \\
A02 & 10350 & 3.55 & SED, H-fit \\
F09 & 10400 & 3.55 & SED, H-fit \\
\hline
\end{tabular}

S81: Sadakane (1981), B82: Boesgaard et al. (1982), A83: Adelman \& Pyper (1983), M85: Morossi \& Malagnini (1985), S93: Smith \& Dworetsky (1993), L98: Landstreet (1998), W00: Wahlgren \& Hubrig (2000), H01: Hubrig \& Castelli (2001), A02: Adelman et al. (2002) F09: this work.

\subsection{Comparison with previous determinations}

Only one previous temperature determination for HD 145788 is known. Glagolevskij (1994) determined a $T_{\text {eff }}$ of $9600 \mathrm{~K}$ from the reddening free $Q$ parameter (Johnson \& Morgan 1953) and of $9100 \mathrm{~K}$ from the $X$ parameter derived from multicolour photometry. The $T_{\text {eff }}$ derived from the $Q$ parameter agrees with our estimation.

The star 21 Peg was analysed for abundances and atmospheric parameters several times in the past. The atmospheric parameters extracted from literature are collected in Table 2, together with the methods of their determination. The last column of Table 2 lists the methods adopted to derive the fundamental parameters for each author. $\mathrm{SPh}$ and $\mathrm{JPh}$ correspond to Strömgren and Johnson photometry respectively. "Fe eq" indicates the use of the Fe ionisation equilibrium, while $\mathrm{H}$-fit the use of hydrogen line fitting. SED corresponds to the use of spectral energy distribution in the visible and/or UV region. All the values obtained from the literature are in excellent agreement with our adopted parameters. We would like to mention that only the oldest determination (Sadakane 1981) was obtained using all the possible parameter indicators, as done in this work.

The spectroscopic literature for $\pi$ Cet is extremely vast and started in the early $60 \mathrm{~s}$. We decided to compare the determinations of $T_{\text {eff }}$ and $\log g$ from the late 70 s on. These data and our determination are presented in Table 3. Our value for $T_{\text {eff }}$ is the only one below $13000 \mathrm{~K}$, while $\log g$ agrees with all the other measurements. Adelman et al. (2002) also derived the parameters of $\pi$ Cet with the simultaneous fitting of $\mathrm{H} \gamma$ and spectrophotometry, essentially the same way as we did. The main difference is that they only used one available spectrophotometric set, while we used all the data found in the literature. The spectrophotometry by Breger (1976) appears a little below the one by Adelman et al. (1989) around the Balmer jump. To be able to simultaneously fit both sets of measurements, we needed a $T_{\text {eff }}$ lower than $13000 \mathrm{~K}$ and the best fit was obtained for $T_{\text {eff }}=12800 \mathrm{~K}$. Also our $\mathrm{H} \beta$ profile is best-fitted only with a $T_{\text {eff }}$ below $13000 \mathrm{~K}$, as already mentioned in Sect. 3.2.1.
Table 3. Atmospheric parameters of $\pi$ Cet derived from other authors.

\begin{tabular}{cccc}
\hline \hline Reference & $\begin{array}{c}T_{\text {eff }} \\
{[\mathrm{K}]}\end{array}$ & $\begin{array}{c}\log g \\
{[\mathrm{cgs}]}\end{array}$ & $\begin{array}{c}\text { Method } \\
\text { (as in Table 2) }\end{array}$ \\
\hline H79 & 13100 & 3.90 & SPh \\
K80 & 13000 & $(\ldots)$ & SED \\
M83 & 13030 & 3.88 & SED(UV) \\
M83 & 13170 & 3.91 & SED(visible) \\
A84 & 13150 & 3.65 & SED H-fit \\
M85 & 13170 & $(\ldots)$ & SED \\
S88 & 13200 & 3.70 & SED \\
M88 & 13425 & $(\ldots)$ & SPh \\
R90 & 13150 & 3.50 & SPh \\
A91 & 13150 & 3.85 & H-fit \\
T91 & 13000 & $(\ldots)$ & SED \\
S92 & 13210 & 3.65 & SPh \\
A02 & 13174 & 3.70 & SPh \\
A02 & 13100 & 3.85 & SED, H-fit \\
F09 & 12800 & 3.75 & SED, H-fit \\
\hline
\end{tabular}

H79: Heacox (1979), K80: Kontizas \& Theodossiou (1980), M83: Malagnini et al. (1983), A84: Adelman (1984), M85: Morossi \& Malagnini (1985), S88: Sadakane et al. (1988), M88: Megessier (1988), R90: Roby \& Lambert (1990), A91: Adelman (1991), T91: Theodossiou \& Danezis (1991), S92: Singh \& Castelli (1992), A02: Adelman et al. (2002), F09: this work. The methods and the acronyms are as in Table 2 .

\section{Abundance analysis}

The VALD database is the main source for the atomic parameters of spectral lines. For light elements C, N, O, Ne I, Mg I, Si II, Si III, S, Ar, and also for Fe III, the oscillator strengths are taken from NIST online database (Ralchenko et al. 2008). LTE abundance analysis in the atmospheres of all three stars is based mainly on the equivalent widths analysed with the improved version of WIDTH9 code updated to use the VALD output linelists and kindly provided to us by Tsymbal.

In the case of blended lines or when the line is situated in the wings of the hydrogen lines, we performed synthetic spectrum calculations with the SyNTh3 code. For our analysis we used the maximum number of spectral lines available in the observed wavelength ranges except lines in spectral regions where the continuum normalisation was too uncertain (high orders of the Paschen series in the ESPaDOnS spectrum of $\pi$ Cet, for example). The final abundances are given in Table 4. A line-by-line abundance list with the equivalent width measurements, adopted oscillator strengths, and their sources is given in Table 9 (online material). Below we discuss the results obtained for individual elements.

\subsection{Results for individual elements}

\subsubsection{Helium}

Stark broadening of helium lines was treated using the Barnard et al. (1974) broadening theory and tables. For allowed isolated lines we used width and shift functions from Table 1 of this paper, while an interpolation of the calculated line profiles given in Tables 2-8 was employed for $\lambda 4472 \AA$ line. All abundances were derived without using equivalent widths, but by fitting of the line wings.

The quality of the fit to the observed He I lines in the programme stars is demonstrated in Fig. 1, while the fit to He I $\lambda 4472 \AA$ line is shown in Fig. 12 for different temperatures in $21 \mathrm{Peg}$ (online material). 
Table 4. LTE atmospheric abundances in programme stars with the error estimates based on the internal scattering from the number of analysed lines, $n$.

\begin{tabular}{|c|c|c|c|c|c|c|c|}
\hline \multirow[t]{2}{*}{ Ion } & \multicolumn{2}{|c|}{ HD 145788} & \multicolumn{2}{|l|}{$21 \mathrm{Peg}$} & \multicolumn{2}{|l|}{$\pi \mathrm{Cet}$} & \multirow{2}{*}{$\begin{array}{c}\text { Sun }(*) \\
\log \left(N / N_{\text {tot }}\right) \\
\end{array}$} \\
\hline & $\log \left(N / N_{\text {tot }}\right)$ & $n$ & $\log \left(N / N_{\text {tot }}\right)$ & $n$ & $\log \left(N / N_{\mathrm{tot}}\right)$ & $n$ & \\
\hline $\mathrm{HeI}$ & $-1.10 \pm 0.05$ & 4 & $-1.09 \pm 0.03$ & 7 & $-0.97 \pm 0.04$ & 6 & -1.12 \\
\hline $\mathrm{C}_{\mathrm{I}}$ & $-3.60 \pm 0.14$ & 5 & $-3.66 \pm 0.14$ & 9 & & & -3.65 \\
\hline C II & $-3.63:$ & 2 & $-3.65 \pm 0.05$ & 4 & $-3.58 \pm 0.07$ & 7 & -3.65 \\
\hline N I & & & $-3.95 \pm 0.12$ & 4 & $-4.03 \pm 0.13$ & 10 & -4.26 \\
\hline N II & & & -3.90 & 1 & $-3.74 \pm 0.07$ & 9 & -4.26 \\
\hline OI & $-3.12 \pm 0.09$ & 7 & $-3.28 \pm 0.11$ & 18 & $-3.06 \pm 0.14$ & 9 & -3.38 \\
\hline O II & & & & & $-3.04:$ & 2 & -3.38 \\
\hline $\mathrm{Ne} I$ & & & $-3.76 \pm 0.05$ & 7 & $-3.66 \pm 0.09$ & 20 & -4.20 \\
\hline $\mathrm{Na}$ & & & $-5.60:$ & 1 & $-5.23 \pm 0.07$ & 3 & -5.87 \\
\hline Mg I & $-4.17 \pm 0.24$ & 5 & $-4.42 \pm 0.12$ & 5 & $-4.27:$ & 2 & -4.51 \\
\hline Mg II & $-4.29 \pm 0.06$ & 4 & $-4.56 \pm 0.03$ & 7 & $-4.47 \pm 0.16$ & 10 & -4.51 \\
\hline $\mathrm{Al} \mathrm{I}$ & -5.70 & 2 & $-5.89:$ & 2 & $-5.57:$ & 2 & -5.67 \\
\hline $\mathrm{Al}$ II & $-5.11 \pm 0.10$ & 3 & $-5.70 \pm 0.10$ & 4 & $-5.73 \pm 0.27$ & 8 & -5.67 \\
\hline $\mathrm{Al}$ III & & & & & $-5.30 \pm 0.02$ & 3 & -5.67 \\
\hline Si I & -4.75 & 1 & $-4.95:$ & 1 & -4.80 & 1 & -4.53 \\
\hline Si II & $-4.27 \pm 0.14$ & 11 & $-4.49 \pm 0.13$ & 22 & $-4.41 \pm 0.20$ & 31 & -4.53 \\
\hline Si III & & & $-4.26 \pm 0.18$ & 2 & $-4.16:$ & 2 & -4.53 \\
\hline P II & & & $-6.37 \pm 0.06$ & 3 & $-6.38 \pm 0.19$ & 9 & -6.68 \\
\hline P III & & & & & $-6.19:$ & 1 & -6.68 \\
\hline S II & $-4.36:$ & 2 & $-4.86 \pm 0.13$ & 26 & $-4.78 \pm 0.16$ & 31 & -4.90 \\
\hline $\mathrm{Cl}$ II & & & & & $-6.95:$ & 2 & -6.54 \\
\hline Ar I & & & & & $-4.86 \pm 0.24:$ & 2 & -5.86 \\
\hline Ar II & & & & & $-5.24 \pm 0.19$ & 6 & -5.86 \\
\hline $\mathrm{Ca} \mathrm{I}$ & $-5.46 \pm 0.11$ & 5 & $-5.84 \pm 0.11$ & 3 & & & -5.73 \\
\hline Ca II & $-5.54 \pm 0.16$ & 6 & $-5.98 \pm 0.08$ & 5 & -5.77 : & 2 & -5.73 \\
\hline Sc II & $-8.90 \pm 0.03$ & 5 & $-9.37 \pm 0.10$ & 7 & $-9.31:$ & 1 & -8.99 \\
\hline Ti II & $-6.80 \pm 0.15$ & 47 & $-7.23 \pm 0.09$ & 59 & $-7.42 \pm 0.08$ & 11 & -7.14 \\
\hline V II & $-7.55 \pm 0.20$ & 4 & $-7.98 \pm 0.06$ & 9 & & & -8.04 \\
\hline CrI & $-6.15 \pm 0.09$ & 4 & $-6.29 \pm 0.09$ & 5 & & & -6.40 \\
\hline Cr II & $-5.86 \pm 0.11$ & 31 & $-6.20 \pm 0.10$ & 68 & $-6.41 \pm 0.10$ & 21 & -6.40 \\
\hline Mn I & $-6.43:$ & 2 & $-6.54 \pm 0.21$ & 5 & & & -6.65 \\
\hline Mn II & $-6.15 \pm 0.08$ & 4 & $-6.51 \pm 0.17$ & 19 & $-6.50 \pm 0.09$ & 3 & -6.65 \\
\hline $\mathrm{Fe} I$ & $-4.23 \pm 0.16$ & 88 & $-4.52 \pm 0.13$ & 108 & $-4.53 \pm 0.22$ & 7 & -4.59 \\
\hline Fe II & $-4.13 \pm 0.15$ & 147 & $-4.50 \pm 0.12$ & 406 & $-4.58 \pm 0.14$ & 186 & -4.59 \\
\hline Fe III & & & $-4.60 \pm 0.06$ & 3 & $-4.52 \pm 0.10$ & 4 & -4.59 \\
\hline Co II & & & $-6.75 \pm 0.18$ & 3 & $-6.93:$ & 1 & -7.12 \\
\hline $\mathrm{Ni}$ I & $-5.32 \pm 0.13$ & 8 & $-5.71 \pm 0.05$ & 10 & & & -5.81 \\
\hline Ni II & $-5.12 \pm 0.05$ & 3 & $-5.61 \pm 0.09$ & 23 & $-5.76 \pm 0.19$ & 17 & -5.81 \\
\hline $\mathrm{ZnI}$ & & & -6.86 & 1 & & & -7.44 \\
\hline Sr II & -8.45 : & 2 & -9.10 & 2 & $-9.15:$ & 2 & -9.12 \\
\hline Y II & $-9.06:$ & 1 & $-9.76 \pm 0.15$ & 4 & & & -9.83 \\
\hline Zr II & $-8.75 \pm 0.32$ & 3 & $-9.48 \pm 0.28$ & 4 & & & -9.45 \\
\hline Ba II & $-8.96 \pm 0.11$ & 3 & $-9.19 \pm 0.06$ & 3 & & & -9.87 \\
\hline $\mathrm{Nd}$ III & & & $-10.09 \pm 0.07$ & 3 & & & -10.59 \\
\hline $\begin{array}{l}T_{\text {eff }} \\
\log g\end{array}$ & $\begin{array}{c}9750 \mathrm{~K} \\
3.7\end{array}$ & & $\begin{array}{c}10400 \mathrm{~K} \\
3.55\end{array}$ & & $\begin{array}{c}12800 \mathrm{~K} \\
3.75\end{array}$ & & $\begin{array}{c}5777 \mathrm{~K} \\
4.44\end{array}$ \\
\hline
\end{tabular}

Internal scattering was not estimated when $n<3$, in which case the derived abundance if flagged with a colon $(:) .(*)$ The abundances of the solar atmosphere calculated by Asplund et al. (2005).

The helium abundance in HD 145788 and in 21 Peg is solar, while it is slightly overabundant in $\pi$ Cet. Our analysis was applied to the He I lines at wavelengths shorter than $5000 \AA$, which should be influenced very little by non-LTE effects, except, maybe, in the case of $\pi$ Cet where LTE synthetic profiles fit the line wings but not the line cores. Non-LTE calculations for He I lines in the spectrum of $\beta$ Ori $\left(T_{\text {eff }}=13000 \mathrm{~K}, \log g\right.$ $=2.0$ ) show that negative non-LTE corrections of about 0.10.2 dex should even be applied to blue lines (Takeda 1994). It is unclear what corrections are expected for main sequence stars of the same $T_{\text {eff }}$, therefore non-LTE calculations for at least $\pi$ Cet are necessary for deriving $\mathrm{He}$ abundance with proper accuracy.
Our high-quality observations of $\pi$ Cet will serve perfectly for a thorough non-LTE study of He I lines in middle B-type stars.

\subsubsection{CNO}

The carbon abundance as derived from C II lines is solar for all three stars. It is also very close to the cosmic abundance standard recently determined by Przybilla et al. (2008) from the analysis of nearby early B-type stars and discussed in their paper. The ionisation equilibrium between C I and C II deserves some short comment. 
Przybilla et al. (2001a) calculated non-LTE corrections for C I and C II for Vega model atmosphere $\left(T_{\text {eff }}=9550 \mathrm{~K}, \log g=\right.$ 3.95). They found these corrections to be negligible. RentzschHolm (1996) made a non-LTE analysis of C II in A-type stars also obtaining very small (less than $0.05 \mathrm{dex}$ ) negative corrections at effective temperatures around $10000 \mathrm{~K}$. Because the ionisation equilibrium between $\mathrm{CI} / \mathrm{C}$ II is fulfilled for HD 145788, we assume that non-LTE corrections are also negligible for this star.

Instead, at higher $T_{\text {eff }}$ values, the abundances obtained from $\mathrm{C}$ I lines having the lower level $3 \mathrm{~s}^{1} \mathrm{P}^{\mathrm{o}}(\lambda \lambda$ 4932, 5052, 5380, $8335,9406 \AA$ ) are significantly lower than those obtained from the other C I lines. In the case of $21 \mathrm{Peg}$, abundances of $\mathrm{CI}$ and $\mathrm{C}$ II only agree if we neglect the abundances obtained from $\lambda$ 4932, 5052, 5380 lines. In the spectrum of the hottest star of our sample, $\pi$ Cet, the situation is even more extreme. $\lambda \lambda 4932$, 5052, $5380 \mathrm{C}$ I lines are not visible at all, while $\lambda \lambda 8335,9406 \AA$ lines appear in emission. The C I lines at $\lambda 7111-7120 \AA$ are rather shallow, we can only determine an upper limit for the abundance: $\log \left(C / N_{\text {tot }}\right)=-4.0$. Like Roby \& Lambert (1990) we also obtain a $\mathrm{CI} / \mathrm{C}$ II imbalance in $\pi$ Cet. Non-LTE calculations by Rentzsch-Holm (1996) seem to explain the unusual behaviour of C I $\lambda \lambda$ 4932, 5052, 5380 lines in stars hotter than $\pi$ Cet because the abundance corrections become positive and grow with the effective temperature.

Nitrogen abundance is obtained for the two hottest stars of our programme from the lines of the neutral and singly-ionised nitrogen. While in $21 \mathrm{Peg}$ we get the evidence for ionisation equilibrium, in $\pi$ Cet $\mathrm{N}$ II lines give higher abundance by 0.3 dex. In both stars, LTE nitrogen abundance exceeds the solar one. For $\pi$ Cet, Roby \& Lambert (1990) derived an approximately solar nitrogen abundance from $\mathrm{N}$ I lines located between two Paschen lines. We use these lines, too, and the higher nitrogen abundance derived by us is caused by the larger equivalent widths, and not by the difference in the adopted effective temperature. From the non-LTE calculations performed by (Przybilla \& Butler 2001), we may expect -0.3 abundance corrections for the lines of $\mathrm{N}_{\mathrm{I}}$ that bring nitrogen abundance in both stars to the solar value. It is difficult to estimate corrections for N II lines. Evidently, nonLTE analysis of both $\mathrm{C} \mathrm{I} / \mathrm{C} \mathrm{II}$ and $\mathrm{N}$ I/N II line formation is necessary.

Oxygen abundance was derived from the lines of neutral oxygen in HD 145788 and in $21 \mathrm{Peg}$, while the lines of neutral and singly-ionised oxygen were used in $\pi$ Cet. Although there are plenty of $\mathrm{O}$ I lines in the red region, our analysis was limited by the lines with $\lambda \leq 6500 \AA$, which are not influenced by non-LTE effects or very little so (Przybilla et al. 2000). Even for $\lambda 6155-8 \AA$, lines the abundance corrections are less than $0.1 \mathrm{dex}$ in main sequence stars. Within the errors of our abundance analysis, 21 Peg has nearly solar oxygen abundance. Moreover, it agrees perfectly with the cosmic abundance standard derived by Przybilla et al. (2008). In HD 145788 and $\pi$ Cet, oxygen seems to be slightly overabundant with values falling in the solar photospheric range defined by Grevesse et al. (1996) and Asplund et al. (2005). For $\pi$ Cet our oxygen abundance agrees perfectly with that obtained by Roby \& Lambert (1990).

\subsubsection{Neon and argon}

The abundance of these noble gases in stellar atmospheres attracts special attention because they cannot be obtained directly in the solar atmosphere. These gases are volatile, and meteoritic studies also cannot provide the actual abundance in the solar system. The revision of the solar abundances by Asplund et al. (2005) that results in a 0.2-0.3 dex decrease in CNO abundances, so those of other elements produced significant inconsistency between the predictions of the solar model and the helioseismology measurements. One of the ways to bring both data into agreement is to increase Ne abundance. Solar model calculations by Bahcall et al. (2005) show that $A(\mathrm{Ne})=8.29 \pm 0.05$ is enough for this purpose.

Cuhna et al. (2006) performed non-LTE analysis of neon line formation in the young B-type stars of the Orion association and derived an average $A(\mathrm{Ne})=8.11 \pm 0.05\left(\log \left(\mathrm{Ne} / N_{\text {tot }}\right)=\right.$ -3.93) from 11 stars. Hempel \& Holweger (2003) derived the $\mathrm{Ne}$ abundance in a sample of optically bright, early B-type main sequence stars, obtaining an average non-LTE Ne abundance of $\log \left(\mathrm{Ne} / N_{\text {tot }}\right)=-3.93 \pm 0.13$. Przybilla et al. (2008) obtained non-LTE Ne abundances in a sample of six nearby main sequence early B-type stars. They derived a Ne abundance of $A(\mathrm{Ne})=8.08 \pm 0.03\left(\log \left(\mathrm{Ne} / N_{\text {tot }}\right)=-3.96\right)$. All these values agree very well with $A(\mathrm{Ne})=8.08 \pm 0.10$ obtained from analysing the emission registered during low-altitude impulsive flare (Feldman \& Widing 1990), but are 0.3 dex higher than adopted by Asplund et al. (2005). Finally, Ne I and Ne II nonLTE analysis in 18 nearby early B-type stars (Morel \& Butler 2008) results in $A(\mathrm{Ne})=7.97 \pm 0.07$. All determinations in B-type stars agree within the quoted errors and provide the reliable estimate of neon abundance in the local interstellar medium, which is higher than the newly proposed solar neon abundance.

Recently, Lanz et al. (2008) have studied the Ar abundance in the same set of young B-type stars of the Orion association and derived an average $A(\mathrm{Ar})=6.66 \pm 0.06\left(\log \left(\mathrm{Ar} / N_{\text {tot }}\right)=\right.$ $-5.38)$, which again agrees with the value $A(\mathrm{Ar})=6.57 \pm 0.12$ obtained by Feldman \& Widing (1990), but is $\sim 0.4$ dex higher than recommended by Asplund et al. (2005). While non-LTE effects on Ne I lines are known to be strong (see Sigut 1999), those on Ar II lines are weak, $\approx 0.03$ dex (Lanz et al. 2008).

We measured Ne I lines in the spectra of $21 \mathrm{Peg}$ and $\pi$ Cet and ArI/Ar II in $\pi$ Cet only. As expected, averaged LTE neon abundances in both stars are higher than the solar one and than that derived by Hempel \& Holweger (2003), Cuhna et al. (2006), Morel \& Butler (2008), or Przybilla et al. (2008) for B-type stars. However, applying non-LTE corrections, calculated by Dworetsky \& Budaj (2000) for the strongest Ne I $6402 \AA$ line to our LTE abundances derived from this line in both stars we get $\log \left(\mathrm{Ne} / N_{\text {tot }}\right)=-3.89$ and -3.86 for 21 Peg and $\pi$ Cet, respectively, which brings $\mathrm{Ne}$ abundance in both stars into rather good agreement with the results obtained for early B-type stars. Rough estimates of possible non-LTE corrections in $\pi$ Cet for Ne I 6402 and $6506 \AA$ lines, for which LTE and non-LTE equivalent widths versus effective temperature are plotted by Sigut (1999), give us $\mathrm{Ne}_{\mathrm{LTE}}-\mathrm{Ne}_{\mathrm{NLTE}} \sim-0.3$ dex for each line, and it agrees with the correction -0.36 calculated by Dworetsky \& Budaj (2000) for Ne I 6402 line. The star $\pi$ Cet is a young star and thus adds reliable current data on the $\mathrm{Ne}$ abundance, taking a large number of high-quality line profiles and secure model atmosphere parameters into account.

We derived argon abundance $\log \left(\mathrm{Ar} / N_{\text {tot }}\right)=-5.24 \pm 0.19$ in $\pi$ Cet from 5 weak but accurately measured Ar II lines. Within error bars this value agrees with the results by Lanz et al. (2008) for B-type stars in the Orion association. We also managed to measure the two strongest Ar I lines at 8103 and $8115 \AA$. They each give an $\mathrm{Ar}$ abundance that is too high. The $8115 \AA$ line is blended with a $\mathrm{Mg}$ II line, and taking this blend into account we get $\log \left(\mathrm{Ar} / N_{\text {tot }}\right)=-5.2$, while the $8103 \AA$ line is too strong 
for its transition probability. Moreover, both lines are located in the region contaminated by weak telluric lines, therefore the extracted abundances may be uncertain.

\subsection{4. $\mathrm{Na}, \mathrm{Mg}, \mathrm{Al}, \mathrm{Si}, \mathrm{P}, \mathrm{S}, \mathrm{Cl}$}

In both 21 Peg and $\pi$ Cet, $\mathrm{Na}$ abundances are derived from lines affected by non-LTE(Takeda 2008), therefore it is not surprising that their measured values are different from the solar ones.

In all three stars we obtained a slight $\mathrm{Mg} \mathrm{I} / \mathrm{Mg}$ II imbalance. For $21 \mathrm{Peg}$ and $\pi$ Cet, the abundance derived from $\mathrm{Mg}$ II lines is close to solar. The abundance derived from the weaker $\mathrm{Mg}$ I lines is consistent to the one obtained from $\mathrm{Mg}$ II, hence solar. We conclude that, for these two stars, $\mathrm{Mg}$ abundance is consistent with the solar one and that discrepancies observed in the strong $\mathrm{Mg}$ I lines come from non-LTE effects.

Magnesium is slightly overabundant in HD 145788, but all the $\mathrm{Mg}$ I lines are affected by non-LTE effects. The sign and the magnitude of its effect depends on both $T_{\text {eff }}$ and $\log g$ (Przybilla et al. 2001b), which prevents us from obtaining any firm conclusion, until detailed non-LTE calculations for Mg I for early Aand middle B-type main sequence stars are carried out.

Aluminum is above solar in HD 145788 and have nearly solar abundances in the two other stars as derived from Al II lines. $\mathrm{Al} \mathrm{I}$ lines in all stars and $\mathrm{Al}$ III lines in $\pi$ Cet provide some discordant results. It is not possible to discuss the ionisation equilibrium without a non-LTE analysis of the line formation of all three ions.

Accurate silicon abundance determination in stars and the interstellar medium is an important part of abundance studies and intercomparisons because $\mathrm{Si}$ is a reference element for meteoritic abundances. Silicon is slightly above solar in HD 145788, and close to solar in $21 \mathrm{Peg}$ and in $\pi$ Cet, if we consider the results obtained from the numerous Si II lines. The spectral synthesis in the region of the only Si I $3905 \AA$ line observed in all three stars provides much lower $\mathrm{Si}$ abundance, and the abundance difference between $\mathrm{Si}$ I and $\mathrm{Si}$ II is practically independent of $T_{\mathrm{eff}}$. A non-LTE analysis of Si line formation in the Sun and in Vega (Wedemeyer 2001) shows that positive non-LTE corrections are expected for Si I $3905 \AA$ line, while small negative corrections may be expected for Si II lines, thus leading both ions into the equilibrium. Our Si analysis is based on the very accurate transition probability for Si I $3905 \AA$ A line (O’Brian \& Lawler 1991) and on a combination of transition probabilities extracted from a recent NIST compilation (Kelleher \& Podobedova 2008) and theoretical calculations by Artru et al. (1981) for Si II lines. The NIST compilation does not contain data for about a quarter of the lines observed in our stars for which rather concordant data exist. Table 8 (online material) gives a collection of the experimental, as well as theoretical, atomic parameters for Si II lines that may be useful in a future non-LTE analysis. The dispersion in the measurements is of the order of the cited accuracies, and theoretical calculations agree rather well with the experimentally measured transition probabilities and Stark widths.

Phosphorus is overabundant relative to the solar abundance by 0.3 dex in both hotter stars, $21 \mathrm{Peg}$ and in $\pi$ Cet. Oscillator strengths for P II lines (taken from VALD) originally come from calculations by Hibbert (1988). Therefore, at least part of the observed overabundance may be caused by uncertainties in calculated transition probabilities. No non-LTE analysis is available for the phosphorus line formation. At the limit of detection, we managed to measure the two strongest $\mathrm{Cl}$ II lines (at $4794,4810 \AA$ ) in $\pi$ Cet. The obtained upper limit on the chlorine abundance is 0.4 dex lower than the recommended solar value (Asplund et al. 2005). Sulphur is overabundant by 0.5 dex in HD 145788 and almost solar in the two other stars.

\subsubsection{Ca and Sc}

These two elements are of special interest in A-type star studies, as their non-solar abundances indicate of a star's classification as a metallic-line (Am) star. In hot Am stars with $T_{\text {eff }}$ close to HD 145788, both elements, in particular scandium, are underabundant by $0.4-0.5 \mathrm{dex}$, while other Fe-peak elements are overabundant by $\sim 0.2-0.3$ dex (see for instance o Peg, which is a typical representative of the hot Am stars, Adelman 1988).

Calcium and scandium are overabundant in HD 145788, but underabundant in $21 \mathrm{Peg}$ by $0.2 \mathrm{dex}$ and $0.4 \mathrm{dex}$, respectively. Formal Ca-Sc classification criteria of Am stars would make 21 Peg the hottest known Am star. However, classical Am stars are also characterised by overabundances of $\sim 0.2-0.3$ dex for other Fe-peak elements, and even more remarkable overabundances of Sr, Y, and Zr (Fossati et al. 2007). All these peculiarities are not observed in $21 \mathrm{Peg}$, which therefore cannot be classified as Am. The star $\pi$ Cet has solar $\mathrm{Ca}$ abundance and the same Sc deficiency as $21 \mathrm{Peg}$.

At the $T_{\text {eff }}$ of 21 Peg non-LTE corrections for both Ca I and $\mathrm{Ca}$ II, lines are expected to be positive (Mashonkina, private communication). In other words, the $\mathrm{Ca}$ abundance obtained from LTE calculations is, perhaps, underestimated, which may explain the observed discrepancies with respect to the solar case. Detailed non-LTE analysis of the formation of $\mathrm{Ca}$ lines is required for accurate abundances.

Including hyperfine splitting ( $h f s$ ) does not change abundance results because $h f s$-effects are negligible for the investigated Sc II lines (see Kurucz' $h f s$ calculations ${ }^{4}$ ). Scandium deficiency requires more careful non-LTE analysis, because it is observed not only in classical Am stars, but also in other stars, for example, in the A-type supergiant Deneb (Schiller \& Przybilla 2008), which have solar abundances of the other Fe-peak elements.

\subsubsection{Ti, V, Mn, Co, Ni}

Within the error limits, all these elements are almost solar in $21 \mathrm{Peg}$. The same is true for $\pi \mathrm{Cet}$, except for Ti, which is slightly underabundant. The $\mathrm{Ti}$ abundance determinations are based on the accurate laboratory transition probabilities (Pickering et al. 2001) currently included in VALD. The non-LTE corrections are expected to be positive (Schiller \& Przybilla 2008), leading to abundance values closer to the solar one.

The situation is a bit different in the atmosphere of HD 145788, where all these elements exhibit 0.2-0.4 dex overabundance relative to the solar photospheric abundances. (This would indicate that the star is Am, but since $\mathrm{Ca}$ and $\mathrm{Sc}$ are not underabundant, the star cannot be classified as Am). Still in HD 145788, for $\mathrm{Mn}, \mathrm{Ni}, \mathrm{Cr}$, and $\mathrm{Fe}$, the lines of the first ions provide slightly higher abundance than the lines of the neutrals, while no significant difference in ionisation equilibrium is observed in the two other stars. The Mn lines are known to have rather large $h f s$. We checked the influence of $h f s$ on the derived Mn abundances for HD 145788 where we measured the largest equivalent widths. Data on $h f s$ for Mn were taken from BlackwellWhitehead et al. (2005) (Mn I) and from Holt et al. (1999)

\footnotetext{
${ }^{4}$ http://cfaku5.cfa.harvard.edu/ATOMS
} 
(Mn II). We found that this effect is weak, and does not exceed 0.05 dex even for the lines with the largest $h f s$.

\subsection{7. $\mathrm{Cr}$}

Although based only on a few lines, abundances derived from $\mathrm{Cr}$ I lines are accurate because the recommended atomic parameters of these transitions (Martin et al. 1988) currently included in VALD are supported by recent precise laboratory measurements (Sobeck et al. 2007).

For CrII, laboratory measurements are only available for the low-lying lines with $E i \leq 4.8 \mathrm{eV}$. Few measured lines in HD 148788 and in $\pi$ Cet, and about one third of $\mathrm{Cr}$ II lines in $21 \mathrm{Peg}$, originated in levels with higher excitation potential. For these lines, only theoretical calculations are available. In several publications, favour was given to the transition probability calculations performed with the orthogonal operator technique (RU: Raassen \& Uylings 1998), which are collected in the RU database ${ }^{5}$ for the Cr II, Fe II, and Co II ions. All these data are included in the current version of VALD. A comparison between RU calculations and the most recent laboratory analysis of 119 lines of $\mathrm{Cr}$ II in 2055-4850 $\AA$ spectral region (Nilsson et al. 2006) shows that both sets agree within $10 \%$ on the absolute scale with a dispersion of 0.13 dex. In the optical spectral region, Nilsson et al. (2006) measures only 7 lines (in 4550$4850 \AA$ region). To provide a consistent analysis, we decided to use RU data for all lines of Cr II in our work. Ionisation equilibrium was found for $21 \mathrm{Peg}$, while a slight imbalance was found between CrI and CrII in HD 145788. In the spectrum of $\pi$ Cet no CrI lines are present.

We found that $\mathrm{Cr}$ is overabundant in HD 145778 by 0.4 dex (as average between the Cr I and Cr II abundances), slightly overabundant in 21 Peg (by $0.15 \mathrm{dex}$ ), while it has solar abundance in $\pi$ Cet.

\subsection{8. $\mathrm{Fe}$}

In $21 \mathrm{Peg}$ and in $\pi$ Cet, iron abundance is practically solar. In particular, the same Fe abundance is derived from Fe lines in three ionisation stage for $\pi$ Cet. No obvious ion imbalance was detected.

Iron is a crucial element for adjusting microturbulence, metallicity, and model atmosphere parameters. It has the most spectral lines in the first three ionisation stages with relatively accurate atomic data that can be observed in the optical spectra of early A- and middle B-type stars. Numerous Fe II lines in the range of excitation energy $2.5-11.3 \mathrm{eV}$ are seen in the spectrum of $21 \mathrm{Peg}$. In the $4000-8000 \AA$ spectral region, laboratory measurements are available for $66 \mathrm{Fe}$ II lines with $E_{\mathrm{i}} \leq 6.2 \mathrm{eV}$ (Ryabchikova et al. 1999). We measured 406 Fe II lines in the 21 Peg spectrum, and more than 200 lines have excitation potential $>10 \mathrm{eV}$. In $\pi$ Cet, 98 out of 186 measured Fe II lines have excitation potential $>10 \mathrm{eV}$. For all these high-excitation lines, atomic parameters are available only through theoretical calculations. As for $\mathrm{Cr}$ II lines, we checked the RU database. For 66 lines for which laboratory transition probabilities are measured, a comparison with the RU data results in $\log g f$ (lab data) $\log g f($ RU data $)=0.11 \pm 0.11$ dex. This means that using the Fe II lines of the homogeneous set of transition probabilities obtained from theoretical calculations and available in the RU database, we may over or underestimate the corresponding abundances by

\footnotetext{
5 ftp://ftp.wins.uva.nl/pub/orth
}

no more than 0.1 dex. Only calculated transition probabilities are available for Fe III lines.

For a comparison of the accuracy of Fe II lines atomic parameters, we obtained three sets of abundance determinations for 21 Peg: one based on laboratory data included in VALD, one based on the recent NIST compilation (Ralchenko et al. 2008), and one based on RU data. The results are (i) from laboratory VALD data: $\log \left(\mathrm{Fe}\right.$ II $\left./ N_{\text {tot }}\right)=-4.61 \pm 0.11$ (51 lines); (ii) from NIST data: $\log \left(\mathrm{Fe}\right.$ II $\left./ N_{\text {tot }}\right)=-4.52 \pm 0.17$ (68 lines); (iii) from the same set of RU data: $\log \left(\mathrm{Fe}\right.$ II $\left./ N_{\text {tot }}\right)=-4.49 \pm 0.09$ (51 lines). Few strong high-excitation Fe II lines are included in the NIST compilation. These results justify the use of RU calculations for accurate iron abundance analysis, when laboratory measurements are not available.

High accuracy of spectral data, together with very low $v \sin i$, and fairly well-established atmospheric parameters, makes 21 Peg a perfect object for an Fe II study. We could measure practically all unclassified lines with intensity 1 and higher given in the list of laboratory measurements (Johansson 1978). These lines belong to the transitions with very high excitation potentials. Accurate position and intensity measurements in stellar spectra may help in further studies of the Fe II spectrum and term system.

Discussion of the possible non-LTE effects on Fe I and Fe II lines was given at the end of Sect. 3.2.2. LTE iron abundances in $21 \mathrm{Peg}$ and in $\pi$ Cet agree well with the cosmic abundance standard (see Przybilla et al. 2008).

\subsubsection{Sr, Y, Zr}

These elements are overabundant in HD 145788 and have solar abundances in $21 \mathrm{Peg}$ and $\pi$ Cet. For the $\mathrm{Zr}$ analysis we used the most recent experimental transition probabilities from Ljung et al. (2006).

\subsubsection{Ba and $\mathrm{Nd}$}

Barium is overabundant in HD 145788 and in 21 Peg. No lines of elements heavier than zirconium are identified in our hottest programme star $\pi$ Cet.

While barium overabundance, together with strontiumyttrium-zirconium overabundances in HD 145788, favours its classification as a hot Am star, barium overabundance in $21 \mathrm{Peg}$, which otherwise has near solar abundances of practically all other elements, is unexpected. Non-LTE corrections to barium abundance, if any, should be positive (L. Mashonkina, private communication). Gigas (1988) derived for Vega non-LTE corrections for the two barium lines $(\lambda \lambda 4554,4934 \AA)$ measured in this work as well. They obtained a positive correction of about 0.3 dex for both lines. The non-LTE corrections that should be applied to the barium abundance obtained in HD 145788 and in 21 Peg make the problem of the barium overabundance even more puzzling. Practically in all the abundance studies of normal A-type stars Ba was found to be overabundant (Lemke 1990; Hill \& Landstreet 1993).

We measured three weak features at the position of the strongest Nd III lines (Ryabchikova et al. 2006) that result in slight $\mathrm{Nd}$ overabundance in $21 \mathrm{Peg}$. While $\mathrm{Nd}$ overabundance may still be attributed to the uncertainties on the absolute scale for calculated transition probabilities or non-LTE effects, which are expected to be negative (Mashonkina et al. 2005), it is not the case for Ba where the atomic parameters of the lines using in our analysis are accurately known from laboratory studies. 


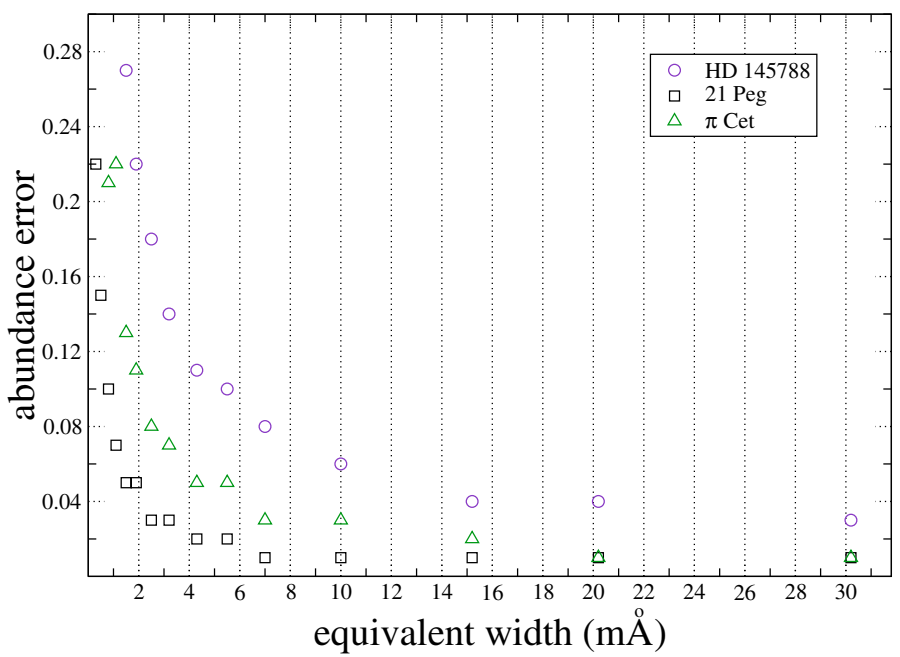

Fig. 5. Error bar in abundance as a function of equivalent widths for HD 145788 (open circle), 21 Peg (open square), and $\pi$ Cet (open triangle). This uncertainty stems from the uncertainty in the equivalentwidth measurement and continuum normalisation. The difference in uncertainty between 21 Peg and $\pi$ Cet comes almost only from the difference in $v \sin i$, while the high error obtained for HD 145788 mainly from the low SNR of our observations, relative to one of the other two stars.

\subsection{Abundance uncertainties}

The abundance uncertainties for each ion shown in Table 4 are the standard deviation of the mean abundance obtained from the individual line abundances. Since our derivation of the abundances is mainly based on equivalent widths, we first have to estimate equivalent width errors given a certain SNR and $v \sin i$. With a two $\sigma$ error bar, we derived $1.2 \mathrm{~m} \AA$ for HD 145788 , $0.2 \mathrm{~m} \AA$ for $21 \mathrm{Peg}$ and $0.5 \mathrm{~m} \AA$ for $\pi$ Cet. These values were derived by assuming a triangular line with a depth (height of the triangulum) equal to $2 \sigma$ (SNR) and a width (base of the triangulum) equal to $2 \times v \sin i$. The rather high uncertainty on the equivalent widths of HD 145788 is mainly due to the low SNR of its spectrum. This shows the importance of a very high SNR not only for fast rotating stars, but also for slowly rotating stars. This uncertainty includes the uncertainty due to the continuum normalisation.

In Fig. 5 we plotted the error bars in abundance for a given line as a function of equivalent widths, for all the three stars analysed in this work. To derive the uncertainty in abundance due to the error bar on equivalent widths measurement, we took a representative Fe II line and derived the abundance of this line on the basis of different values of equivalent widths ranging from $0.3 \mathrm{~m} \AA$ to $110 \mathrm{~m} \AA$. We then calculated the difference between the abundance obtained with the equivalent width $\mathrm{X}$ and $\mathrm{X}+\delta \mathrm{X}$, where $\delta \mathrm{X}$ is the error bar on the equivalent widths measurement. As expected, HD 145788 is the star that shows the largest error bar. For equivalent widths greater than $30 \mathrm{~m} \AA$, the abundance error tends asymptotically to zero. Table 9 also shows that all the lines measured for this work are above the detection limit given by the $v \sin i$ and the SNR.

The mean equivalent width measured in these three stars is about $20 \mathrm{~m} \AA$ for HD $145788,5 \mathrm{~m} \AA$ for $21 \mathrm{Peg}$, and $8 \mathrm{~m} \AA$ for $\pi$ Cet. These values correspond to an error bar in abundance, because of the uncertainty on the equivalent widths measurement and continuum normalisation of $0.04 \mathrm{dex}$ for HD 145788, and 0.03 dex for both $21 \mathrm{Peg}$ and $\pi$ Cet.

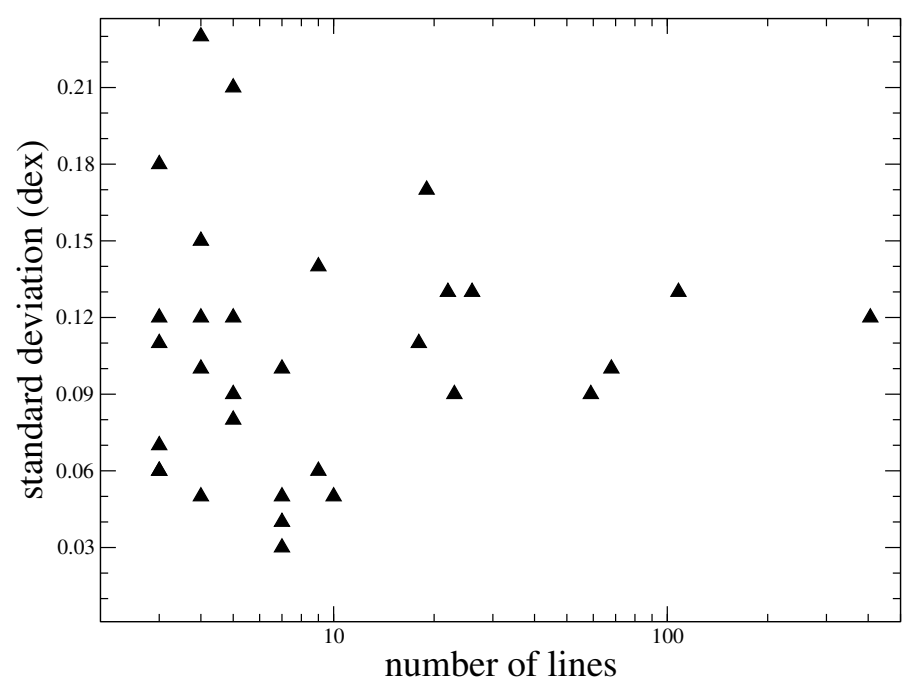

Fig. 6. Standard deviation of the derived abundances as a function of the the number of lines (shown in logarithmic scale and for a number of lines greater than 2). For visualisation reasons we omitted the standard deviation given by $\mathrm{Zr}$ II.

When we have enough measured lines, we assume that the internal scatter for each ion also takes the errors due to equivalent widths measurement and continuum normalisation into account.

Figure 6 shows the abundance scatter as a function of the number of measured lines for 21 Peg. For elements where nonLTE effects are supposed to be important and line-dependent, such as $\mathrm{Al}$ and $\mathrm{S}$, the internal standard deviation is particularly high. The same is found for elements with lower accuracy in $\log g f$ values due to the complexity of the atomic levels, such as Si. For other elements with a large enough number of spectral lines, say $n>10$, it is reasonable to expect an internal error of 0.11 dex (see Fig. 6).

Considering the errors in oscillator strengths determination (see Table 9) given for the laboratory data, we may say that these errors are smaller for most elements than the internal scatter, so do not significantly influence the final results. The same is relevant for theoretical calculations. As already shown for Cr II and Fe II (Sect. 4.1.8), calculated and laboratory sets of oscillator strengths agree within 0.1 dex.

It should be noted that error due to the internal scatter is just a part of the total error bar on the abundance determination. To derive a more realistic abundance uncertainty we also have to take the error bar due to systematic uncertainties in the fundamental parameters into account. For a detailed discussion of the abundance error bars we again use $21 \mathrm{Peg}$. Table 5 shows the variation in abundance for each analysed ion, caused by the change of one fundamental parameter by $+1 \sigma$, keeping fixed the other parameters. For the comparison $\sigma_{\text {abn }}^{2}$ (syst.) with $\sigma_{\text {abn }}$ (scatt.) of those ions for which the internal scattering could not be measured, an a priori scatter of $\sigma_{\text {abn }}$ (scatt.) $=0.11$ dex has to be assumed.

The main source of uncertainty is the error in the effective temperature determination, while the variation due to $\log g$ and, in particular, to $v_{\text {mic }}$ is almost negligible, although here we are considering an uncertainty on $v_{\text {mic }}$ of $2 \sigma$. The abundance variation due to an increase in $\log g$ leads to a worse ionisation equilibrium for many elements for which the equilibrium is reached at the adopted $\log g$, such as $\mathrm{Cr}, \mathrm{Mn}, \mathrm{Fe}$, and $\mathrm{Ni}$. We repeated the same analysis for Fe II for HD 145788 and $\pi$ Cet. The results 
Table 5. Error sources for the abundances of the chemical elements of 21 Peg.

\begin{tabular}{|c|c|c|c|c|c|c|}
\hline 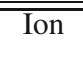 & $\begin{array}{l}\text { Abundance } \\
\log \left(N / N_{\text {tot }}\right)\end{array}$ & $\begin{array}{c}\left.\sigma_{\text {abn }} \text { (scatt. }\right) \\
(\text { dex })\end{array}$ & $\begin{array}{c}\sigma_{\text {abn }}\left(T_{\text {eff }}\right) \\
(\operatorname{dex})\end{array}$ & $\begin{array}{c}\sigma_{\mathrm{abn}}(\log g) \\
(\operatorname{dex})\end{array}$ & $\begin{array}{c}\sigma_{\text {abn }}\left(v_{\text {mic }}\right) \\
(\text { dex })\end{array}$ & $\begin{array}{c}\sigma_{\text {abn }}(\text { syst. }) \\
(\text { dex })\end{array}$ \\
\hline $\mathrm{He} \mathrm{I}$ & -1.11 & 0.04 & -0.08 & 0.03 & 0.00 & 0.09 \\
\hline C I & -3.66 & 0.14 & -0.05 & -0.13 & -0.10 & 0.17 \\
\hline C II & -3.65 & 0.05 & -0.17 & -0.02 & -0.08 & 0.19 \\
\hline $\mathrm{N}_{\mathrm{I}}$ & -3.95 & 0.12 & -0.05 & -0.07 & -0.05 & 0.10 \\
\hline $\mathrm{N}_{\text {II }}$ & -3.90 : & & -0.09 & 0.05 & 0.00 & 0.10 \\
\hline O I & -3.28 & 0.11 & 0.00 & -0.02 & -0.02 & 0.03 \\
\hline $\mathrm{Ne} I$ & -3.76 & 0.05 & -0.18 & -0.03 & -0.06 & 0.19 \\
\hline $\mathrm{Na} \mathrm{I}$ & -5.60 & & 0.04 & -0.04 & -0.01 & 0.06 \\
\hline Mg I & -4.42 & 0.12 & 0.10 & -0.06 & -0.05 & 0.13 \\
\hline Mg II & -4.56 & 0.03 & -0.02 & -0.01 & -0.02 & 0.03 \\
\hline $\mathrm{Al} \mathrm{I}$ & -5.89 & & 0.10 & -0.03 & 0.00 & 0.10 \\
\hline Al II & -5.68 & 0.10 & -0.09 & 0.01 & -0.03 & 0.10 \\
\hline $\mathrm{Si}$ I & -4.95 & & 0.10 & -0.02 & 0.00 & 0.10 \\
\hline Si II & -4.49 & 0.13 & -0.07 & 0.01 & -0.03 & 0.08 \\
\hline Si III & -4.26 & 0.18 & -0.16 & 0.04 & -0.03 & 0.17 \\
\hline P II & -6.37 & 0.06 & -0.08 & 0.02 & -0.02 & 0.08 \\
\hline S II & -4.86 & 0.13 & -0.14 & 0.00 & -0.05 & 0.15 \\
\hline $\mathrm{Ca} \mathrm{I}$ & -5.84 & 0.11 & 0.20 & -0.07 & 0.00 & 0.21 \\
\hline Ca II & -5.98 & 0.08 & 0.03 & -0.05 & -0.04 & 0.07 \\
\hline Sc II & -9.37 & 0.10 & 0.12 & -0.01 & 0.00 & 0.12 \\
\hline Ti II & -7.23 & 0.09 & 0.08 & 0.00 & -0.02 & 0.08 \\
\hline V II & -7.98 & 0.06 & 0.06 & 0.02 & 0.00 & 0.06 \\
\hline $\mathrm{Cr} \mathrm{I}$ & -6.29 & 0.09 & 0.13 & -0.03 & 0.00 & 0.13 \\
\hline Cr II & -6.20 & 0.10 & 0.03 & 0.02 & -0.01 & 0.04 \\
\hline Mn I & -6.54 & 0.21 & 0.14 & -0.03 & 0.00 & 0.14 \\
\hline Mn II & -6.51 & 0.17 & 0.01 & 0.01 & -0.01 & 0.01 \\
\hline $\mathrm{Fe} I$ & -4.52 & 0.13 & 0.11 & -0.03 & 0.00 & 0.11 \\
\hline $\mathrm{Fe}$ II & -4.50 & 0.12 & -0.02 & 0.02 & -0.02 & 0.03 \\
\hline $\mathrm{Fe}$ III & -4.60 & 0.06 & -0.10 & 0.06 & -0.01 & 0.12 \\
\hline Co II & -6.75 & 0.18 & 0.01 & 0.03 & 0.00 & 0.03 \\
\hline $\mathrm{Ni}$ I & -5.71 & 0.05 & 0.09 & -0.03 & -0.01 & 0.10 \\
\hline Ni II & -5.61 & 0.09 & -0.04 & 0.03 & -0.01 & 0.05 \\
\hline $\mathrm{ZnI}$ & -6.86 & & 0.10 & -0.02 & 0.00 & 0.10 \\
\hline Sr II & -9.10 & 0.01 & 0.13 & -0.03 & -0.06 & 0.15 \\
\hline Y II & -9.76 & 0.15 & 0.13 & -0.02 & -0.01 & 0.13 \\
\hline Zr II & -9.48 & 0.28 & 0.11 & 0.00 & 0.00 & 0.11 \\
\hline Ba II & -9.19 & 0.06 & 0.14 & -0.02 & -0.01 & 0.14 \\
\hline Nd III & -10.09 & 0.07 & 0.04 & 0.04 & 0.00 & 0.06 \\
\hline
\end{tabular}

Column 3 standard deviation $\sigma_{\text {abn }}$ (scatt.) of the mean abundance obtained from different spectral lines (internal scattering); a blank means that the number of spectral lines is $<3$, hence no internal scattering could be estimated. (Note that these values are identical to those given in Table 4.) Columns 4-6 give the variation in abundance estimated by increasing $T_{\text {eff }}$ by $200 \mathrm{~K}, \log g$ by 0.1 dex, and $v_{\text {mic }}$ by $0.4 \mathrm{~km} \mathrm{~s}^{-1}$, respectively. Column 7 gives the the mean error calculated applying the standard error propagation theory on the systematic uncertainties given in Col. 4-6, i.e., $\sigma_{\text {abn }}^{2}($ syst. $)=\sigma_{\text {abn }}^{2}\left(T_{\text {eff }}\right)+\sigma_{\text {abn }}^{2}(\log g)+\sigma_{\text {abn }}^{2}\left(v_{\text {mic }}\right)$.

are comparable with those obtained for $21 \mathrm{Peg}$. We also expect the same effect for the other ions.

Assuming the different errors in the abundance determination are independent, we derived the final error bar using standard error propagation theory, given in column six of Table 5. When the abundance is given by a single line, we assumed an internal error of $0.11 \mathrm{dex}$. Using the propagation theory we considered the situation where the determination of each fundamental parameter is an independent process. The mean value of the LTE uncertainties given in column six of Table 5 is 0.16 dex.

Because of the high SNR of the observations, the low $v \sin i$ and the non peculiarity of the programme stars, we can reasonably believe that the errors in abundance determinations estimated in the present study are the smallest ones that could be obtained with the current state of the art of spectral LTE analysis for early-type stars. In the cases of stars with $v \sin i$ higher than those considered in this work, the uncertainty on the abundances increases. A higher rotational velocity would force the abundance analysis to be based on strong and saturated lines that are more sensitive to $v_{\text {mic }}$ variations than weak lines. For a more detailed discussion, see Fossati et al. (2008).

\subsection{Comparison with previous abundance determinations}

Table 6 (online) collects all previous massive abundance determinations in the atmosphere of 21 Peg (Sadakane 1981; Smith \& Dworetsky 1993; Smith 1993, 1994; Dworetsky \& Budaj 2000) in comparison with the results of the current analysis. We do not include works that only give abundances for a $\mathrm{Cr}$ and/or Fe.

The main advantage of our analysis over the previous ones is the wide wavelength coverage and the high quality of our spectra. It allows us to use many more spectral lines including very weak ones of the species not analysed before. Our analysis provides homogeneous abundance data for 38 ions of 26 chemical elements from $\mathrm{He}$ to $\mathrm{Nd}$. 


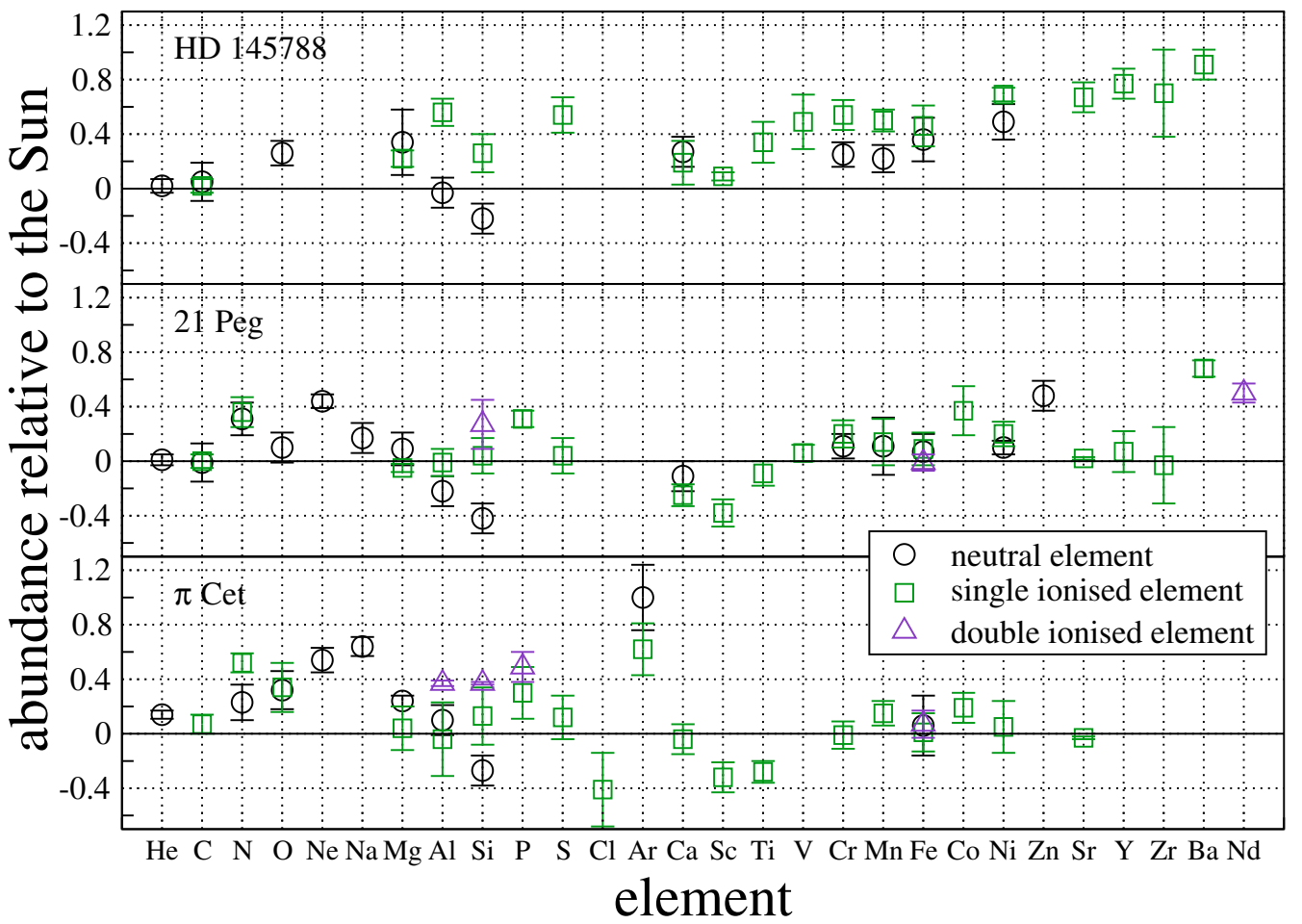

Fig. 7. LTE abundances relative to the Sun (Asplund et al. 2005) for HD 145788, $21 \mathrm{Peg}$, and $\pi$ Cet, from top to bottom. The open circles, squares, and triangles indicate the abundance for the neutral element, single ionised, and double ionised, respectively. The possible non-LTE corrections for each ion are described in the text from Sects. 4.1.1 to 4.1.10.
Reasonably good agreement exists between our abundances derived with the spectra in optical and IR spectral regions and those derived with the UV observations (Smith \& Dworetsky 1993; Smith 1993, 1994), supporting the correctness of the adopted model atmosphere.

Dworetsky \& Budaj (2000) derived the LTE neon abundance and gave non-LTE abundance corrections for the strongest $\mathrm{Ne}$ I $\lambda 6402$ line. If we apply the non-LTE correction described in Sect. 4.1.3 to this line, we almost get the same non-LTE abundance.

For $\pi$ Cet the number of abundance determinations in the literature is particularly vast. As for $21 \mathrm{Peg}$ we consider only those where abundances are derived for large number of ions: Adelman (1991), Smith \& Dworetsky (1993), Smith (1993), Smith (1994), and Acke \& Waelkens (2004), except for neon abundance where we again included LTE and non-LTE results by Dworetsky \& Budaj (2000). For the comparison (see the online Table 7) of the abundances obtained for $\pi$ Cet, we also show the adopted $v_{\text {mic }}$ because we believe that differences in this parameter are responsible for the difference between our abundances and those of Acke \& Waelkens (2004). Again, we emphasise that the total number of ions (36) and elements (22), as well as the number of lines per ion analysed in the present work, is much higher than in any previous study. For $\mathrm{Al}, \mathrm{Si}, \mathrm{Fe}$, we managed to derive abundances from the lines of the element in three ionisation stages, which provides a unique possibility to study non-LTE effects.

For the ions having several lines (S II, Ti II, Cr II and Fe II) we generally get a good agreement among the different authors, in particular, for Fe II. The lower abundances given by Acke \& Waelkens (2004) are due to the high $v_{\text {mic }}$ adopted.

The difference in He abundance between our work and that by Adelman (1991) may be explained by the difference in $T_{\text {eff }}$, already discussed in Sect. 3.4. Both LTE and non-LTE neon abundances agree rather well with the results by Dworetsky \& Budaj (2000) after applying the non-LTE corrections given by these authors.

\section{Discussion}

In Fig. 7 we show the derived abundances normalised to solar values (Asplund et al. 2005). In the following, we discuss the stars of our sample individually.

\subsection{HD 145788}

HD 145788 shows a slight overabundance for almost all ions and typical Am abundance pattern for elements heavier than Ti. The overall overabundance and the Am abundance pattern could be explained if HD 145788 was formed in a region of the sky with a metallicity higher than the solar region. To be able to check the possible Am classification of HD 145788, we can compare the obtained abundance pattern with the typical one for Am stars in clusters having enhanced metallicity, e.g. the Praesepe open cluster with an overall metallicity of $[\mathrm{Fe} / \mathrm{H}]=0.14 \mathrm{dex}$ (Chen et al. 2003). Fossati et al. (2007) give the abundance pattern of eight Am stars belonging to the Praesepe cluster. All these stars show clear Am signatures: underabundances of CNO and Sc, and overabundances of the elements heavier than Ti. In HD 145788 the underabundances of $\mathrm{CNO}$ and $\mathrm{Sc}$ are not visible. For this reason we believe that the star cannot be classified as a hot Am star and that the observed abundance pattern stems from the composition of the cloud where HD 145788 was formed.

Table 4 and Fig. 7 show that with the adopted fundamental parameters of HD 145788 we get ionisation imbalance for the Fe-peak elements. With a small adjustment of the parameters within the error bars $\left(T_{\text {eff }}:+100 \mathrm{~K}, \log g:-0.05\right)$ it is possible to compensate for this imbalance for the Fe-peak elements, but the ionisation balance becomes worse for other elements such as $\mathrm{C}, \mathrm{Mg}$, and $\mathrm{Ca}$. This is a clear sign that a non-LTE analysis is needed for this object to understand whether the ionisation violation stems from non-LTE effects or to some other physical effect. Unfortunately we cannot be completely certain of the adopted parameters because of the lack of spectrophotometric measurements. 


\section{2. $21 \mathrm{Peg}$}

The star 21 Peg shows solar abundances for almost all elements. At the temperature of $21 \mathrm{Peg}$, slowly rotating stars are usually hot Am, cool HgMn, or magnetic stars.

The possibility of classifying $21 \mathrm{Peg}$ as an Am star (which is potentially suggested by the observed Sc underabundance) is excluded by the solar abundances of all the other mentioned indicators. As explained in Sect. 4.1.5, the non-LTE correction for $\mathrm{Ca}$ is expected to be positive, increasing the $\mathrm{Ca}$ abundance to the solar value, but detailed non-LTE analysis should be performed for a more accurate determination. Almost nothing is known about non-LTE effects for Sc at these temperatures, so that to understand whether the observed underabundance is real, a non-LTE analysis should be performed.

Caliskan \& Adelman (1997), Adelman (1998), Adelman (1999), and Kocer et al. (2003) have published abundances of several chemically normal, early-type stars. The derived Sc abundance for stars with an effective temperature similar to that of 21 Peg is almost always below the solar value, with nearly solar abundances for other Fe-peak elements. We thus conclude that the observed abundances of most elements but $\mathrm{Ba}$ in $21 \mathrm{Peg}$ allow us to classify it as a normal early-type rather than Am star. Solar Mn abundance and the absence of Hg II $\lambda 3984 \AA$ line exclude any classification of $21 \mathrm{Peg}$ as an $\mathrm{HgMn}$ star.

\section{3. $\pi$ Cet}

The star $\pi$ Cet shows solar abundances for almost all elements. Only $\mathrm{O}, \mathrm{Ne}, \mathrm{Na}$ and $\mathrm{Ar}$ are clearly overabundant. For $\mathrm{Ne}$ or $\mathrm{Na}$ this most probably comes from non-LTE effects. For argon, the non-LTE effects are expected to be weak (see Sect. 4.1.3). In other chemically normal, early-type stars the Ar abundance appears to be above the solar one (see Lanz et al. 2008; Fossati et al. 2007; Adelman 1998), leading to the conclusion that indirect solar Ar abundance given by Asplund et al. (2005) is underestimated.

As for $21 \mathrm{Peg}$, the Sc underabundance of $\pi$ Cet is not an indication of a possible Am peculiarity. The absence of magnetic field or of normal $\mathrm{Mn}$ abundance, including no trace of any $\mathrm{Hg}$ signature in the spectrum exclude a classification of the star as magnetic peculiar or non-magnetic $\mathrm{HgMn}$ object.

The star $\pi$ Cet is a known binary with a period of about 7.5 years (Lacy et al. 1997) and is a Herbig AeBe star (Malfait et al. 1998). The Herbig classification comes from a detected infrared excess at wavelengths longer than $10 \mu \mathrm{m}$. The two spectra obtained with ESPaDOnS show variability in the line profiles, small emission-like features close to the core of $\mathrm{H} \alpha$, and emission features at the position of C I $\lambda \lambda \sim 8335$ and $9405 \AA$ in the near infrared. The width of $\mathrm{CI}$ emission lines are exactly the same as expected for absorption line in $\pi$ Cet. The pre-mainsequence status of this star, which is very likely responsible for these emissions, might also explain the variability observed in the spectral lines, as circumstellar absorption or emission coming from a proto-planetary disk. The variation in the line profile within one day excludes the possibility that the observed changes are caused by the companion.

Another explanation for the line variations comes from pulsation. We performed a frequency analysis of the radial velocity measurements given by Lacy et al. (1997) and the ones obtained from the two ESPaDOnS spectra. Preliminary results show that two frequencies appear in the amplitude spectrum: one corresponding to the orbital period and another one at $\sim 2.79$ day $^{-1}$. This frequency is consistent with the expected pulsation periods for SPB stars with effective temperature of $\pi$ Cet (see Fig. 5 of Pamyatnykh 1999). In this way pulsation could explain the lineprofile variation, while the presence of a disk around the star could explain the small emission visible in $\mathrm{H} \alpha$. The $\mathrm{C}$ I emission lines in the near infrared could be explained either by the disk or by non-LTE effects (Nieva \& Przybilla 2008).

Only future photometric observations and time-resolved spectroscopy and non-LTE analysis could lead to a better understanding of the star's status.

\section{Conclusions: are solar abundances also a reference for early A- and late B-type stars?}

One of the main goal of this work was to check whether the solar abundances can be taken as a reference for early-type stars. The same question has recently been discussed by Przybilla et al. (2008) who analysed a sample of early B-type stars in the solar neighbourhood to compare the obtained non-LTE abundances with the ones published by other authors for stars in the Orion nebula, various B-type stars, young F- and G-type stars, the interstellar medium, and the sun (Grevesse et al. 1996; Asplund et al. 2005), and to check the chemical homogeneity of the solar neighbourhood. They obtained an excellent agreement between the non-LTE abundances for $\mathrm{He}, \mathrm{C}, \mathrm{N}, \mathrm{Mg}, \mathrm{Si}$, and Fe with the solar ones published by Asplund et al. (2005), while the oxygen abundance lies between the solar values obtained by Grevesse et al. (1996) and Asplund et al. (2005), and the Ne abundance is compatible with the one provided by Grevesse et al. (1996).

The optical spectra of early B-type stars cannot provide reliable data for many other elements (Ca, Ti, Cr, Mn, Sr, Y, Zr), which are important for comparative abundance studies of chemically peculiar stars. While early A- and late B-type stars, investigated in the present paper, provide us with the abundances of up to 26 elements, most of which are based on enough spectral lines with accurately known atomic parameters. Figure 7 shows almost solar abundances for many elements in both 21 Peg and $\pi$ Cet, while the observed abundance pattern in HD 145788 gives a hint that the star may have been formed in a region of the sky at high metallicity. In early-type stars it is possible to directly derive the He abundance, while for the Sun it is only possible through astroseismological observations and modelling. For this reason it is important to check that the He abundance is comparable to the solar value in several chemically normal early-type stars.

In the analysed stars, several elements $(\mathrm{He}, \mathrm{C}, \mathrm{Al}, \mathrm{S}, \mathrm{V}, \mathrm{Cr}$, $\mathrm{Mn}, \mathrm{Fe}, \mathrm{Ni}, \mathrm{Sr}, \mathrm{Y}, \mathrm{Zr}$ ) show abundances compatible with the revised solar data (Asplund et al. 2005), and when discrepancies are present they could be explained by non-LTE effects (N, Na, $\mathrm{Mg}, \mathrm{Si}, \mathrm{Ca}, \mathrm{Ti}, \mathrm{Nd})$. For $\mathrm{Ne}$ and Ar the expected non-LTE corrections would lead to abundances close to those derived for early B-type stars (Lanz et al. 2008; Przybilla et al. 2008) or to the solar ones given by Grevesse et al. (1996) instead of Asplund et al. (2005). Non-LTE corrections were never calculated and should be determined for other elements that show differences with the solar abundance $(\mathrm{P}, \mathrm{Cl}, \mathrm{Sc}, \mathrm{Co})$. We found actual discrepancies with the solar abundance for oxygen in $\pi$ Cet and for $\mathrm{Ba}$ in $21 \mathrm{Peg}$. While the oxygen problem may be solved by careful non-LTE analysis of all the available lines including the red and IR ones, the Ba overabundance cannot be explained by the current non-LTE results. The abundances obtained in this work for this set of three early B-type stars agree very well with the ones obtained by Przybilla et al. (2008) for all the elements.

The published abundances of $\mathrm{Ba}$ in chemically normal early-type stars (Lemke 1990; Caliskan \& Adelman 1997; 
Adelman 1999; Kocer et al. 2003) show a definite trend towards a Ba overabundance. The non-LTE corrections for Ba should be positive, leading to an even greater discrepancy with the solar value, that probably does not represent early-type stars.

Non-LTE effects are studied mainly in solar-type stars, lowmetallicity stars, and giants, and in stars hotter than early B-type, where the effects are expected to be strong. Very few analyses have been performed for normal early A- and late B-type stars (e.g. Vega), and our study claims the real need of such analyses for many elements before making a definite conclusion about the solar abundances as standards for early-type stars.

Acknowledgements. This work is based on observations collected at the Nordic Optical Telescope (NOT) as part of programme number 35-001 and at the ESO $3.6 \mathrm{~m}$ telescope at Cerro La Silla (Chile). Part of this work is based on observations made with the Nordic Optical Telescope, operated on the island of La Palma jointly by Denmark, Finland, Iceland, Norway and Sweden, in the Spanish Observatorio del Roque de los Muchachos of the Instituto de Astrofisica de Canarias. This work is also based on observations obtained at the Canada-France-Hawaii Telescope (CFHT), which is operated by the National Research Council of Canada, the Institut National des Sciences de l'Univers of the Centre National de la Rechereche Scientifique of France, and the University of Hawaii. This work is supported by the Austrian Science Foundation (FWF project P17890-N2 - LF, TR and OK), by the Russian Foundation for Basic research (grant 08-02-00469a - TR) and by the Presidium RAS Programme "Origin and evolution of stars and galaxies" (TR). G.A.W. acknowledges support from the Academic Research Programme (ARP) of the Department of National Defence (Canada). We thank D. Lyashko for having developed and provided the reduction pipeline for the FIES data, V. Tsymbal for providing us with an improved version of WIDTH9, and L. Mashonkina for providing some non-LTE estimates. We thank the anonymous referee for the constructive comments. We thank A. Ederoclite and L. Monaco for the spectrum of HD 145788, and M. Gruberbauer for the frequency analysis. T.R. and L.F. thank D. Shulyak for the fruitful help, support and discussion of model atmospheres and spectral energy distribution. This work made use of the MAST-IUE archive (http://archive.stsci.edu/iue/), of SAO/NASA ADS, SIMBAD, VIZIER and of the VOSpec tool (http://www.euro-vo.org/pub/fc/software.html) developed for the European Virtual Observatory. This publication makes use of data products from the Two Micron All Sky Survey, which is a joint project of the University of Massachusetts and the Infrared Processing and Analysis Center/California Institute of Technology, funded by the National Aeronautics and Space Administration and the National Science Foundation.

\section{References}

Acke, B., \& Waelkens, C. A\&A, 2004, 427, 1009

Adelman, S. J. 1984, A\&AS, 55, 479

Adelman, S. J. 1988, MNRAS, 230, 671

Adelman, S. J. 1991, MNRAS, 252, 116

Adelman, S. J. 1998, MNRAS, 296, 856

Adelman, S. J. 1999, MNRAS, 310, 146

Adelman, S. J., \& Pyper, D. M. 1983, ApJ, 266, 732

Adelman, S. J., \& Gulliver, A. F. 1990, ApJ, 348, 712

Adelman, S. J., \& Unsuree, N. 2007, Balt. Astron., 16, 183

Adelman, S. J., Pyper, D. M., Shore, S. N., White, R. E., \& Warren, Jr., W. H. 1989, A\&AS, 81, 221

Adelman, S. J., Pintado, O. I., Nieva, M. F., Rayle, K. E., \& Sanders, Jr., S. E. 2002, A\&A, 392, 1031

Artru, M.-C., Jamar, C., Petrini, D., \& Praderie, F. 1981, A\&A, 96, 380

Asplund, M., Grevesse, N., \& Sauval, A. J. 2005, ASP Conf. Ser., 336, 25

Auer, L. H., Mihalas, D., Aller, L. H., \& Ross, J. E. 1966, ApJ, 145, 153

Bagnulo, S., Wade, G. A., Donati, J.-F., et al. 2001, A\&A, 369, 889

Bahcall, J. N., Basu, S., \& Serenelli, A. M. 2005, ApJ, 631, 1281

Barach, J. P. 1970, JQSRT, 10, 519

Bard, A., \& Kock, M. 1994, A\&A, 282, 1014

Bard, A., Kock, A., \& Kock, M. 1991, A\&A, 248, 315

Barklem, P. S., Piskunov, N., \& O’Mara, B. J. 2000, A\&A, 363, 1091

Barnard A. J., Cooper J., \& Smith E. W. 1974, JQSRT, 14, 1025

Bengston, R. D., \& Miller, M. H. 1970, JOSA, 60, 1093

Berry, H. G., Bromander, J., Curtis, L. J., \& Buchta, R. 1971, Phys. Scr., 3, 125

Biémont E., Grevesse N., Faires L. M., et al. 1989, A\&A, 209, 391

Bizzarri, A., Huber, M. C. E., Noels, A., et al. 1993, A\&A, 273, 707

Blackwell, D. E., Booth, A. J., Petford, A. D., \& Laming, J. M. 1989, MNRAS, 236,235
Blackwell-Whitehead, R. J., Pickering, J. C., \& Pearse, O. 2005, ApJS, 157, 402 Blanco, F., Botho, B., \& Campos, J. 1995, Phys. Scr., 52, 628

Boesgaard, A. M., Heacox, W. D., Wolff, S. C., Borsenberger, J., \& Praderie, F. 1982, ApJ, 259, 723

Breger, M. 1976, ApJS, 32, 7

Caliskan, H., \& Adelman, S. J. 1997, MNRAS, 288, 501

Chen, L., Hou, J. L., \& Wang, J. J. 2003, AJ, 125, 1397

Cunha, K., Hubeny, I., \& Lanz, T. 2006, ApJ, 647, L143

Cutri, R. M., Skrutskie, M. F., van Dyk, S., et al., 2003, 2MASS All Sky Catalog of point sources, The IRSA 2MASS All-Sky Point Source Catalog, NASA/IPAC Infrared Science Archive

Donati, J.-F., Semel, M., Carter, B. D., Rees, D. E., \& Collier Cameron, A. 1997, MNRAS, 291, 658

Dworetsky, M. M., \& Budaj, J. 2000, MNRAS, 318, 1264

Fekel, F. C. 2003, PASP, 115, 807

Feldman, U., \& Widing, K. G. 1990, ApJ, 363, 292

Fossati, L., Bagnulo, S., Monier, R., et al. 2007, A\&A, 476, 911

Fossati, L., Bagnulo, S., Landstreet, J., et al. 2008, A\&A, 483, 891

Fuhr, J. R., Martin, G. A., \& Wiese, W. L. 1988. J. Phys. Chem. Ref. Data, 17, Suppl. 4

Gigas, D. 1986, A\&A, 165, 170

Gigas, D. 1988, A\&A, 192, 264

Glagolevskij, Y. V. 1994, Bull. Special Astrophys. Obs., 38, 152

Gregg, M. D., Silva, D., Rayner, J., et al. 2004, BAAS, The HST/STIS Next Generation Spectral Library, 36, 1496

Grevesse, N., Noels, A., \& Sauval, A. J. 1996, ASP Conf. Ser., 99, 117

Hannaford, P., Lowe, R. M., Grevesse, N., Biemont, E., \& Whaling, W. 1982, ApJ, 261, 736

Hauck, B., \& Mermilliod, M. 1998, A\&AS, 129, 431

Heacox, W. D. 1979, ApJS, 41, 675

Hibbert, A. 1988, Phys. Scr., 38, 37

Hill, G. M., \& Landstreet, J. D. 1993, A\&A, 276, 142

Hempel, M., \& Holweger, H. 2003, A\&A, 408, 1065

Holt, R. A., Scholl, T. J., \& Rosner, S. D. 1999, MNRAS, 306, 107

Hubrig, S., \& Castelli, F. 2001, A\&A, 375, 963

Hubrig, S., Yudin, R. V., Schöller, M., \& Pogodin, M. A. 2006, A\&A, 446, 1089

Jamar, C., Macau-Hercot, D., Monfils, A., et al. 1976, Ultraviolet bright-star spectrophotometric catalogue, A compilation of absolute spectrophotometric data obtained with the Sky Survey Telescope (S2/68) on the European Astronomical Satellite TD-1

Johansson, S. 1978, Phys. Scr., 18, 217

Johnson, H. L., \& Morgan, W. W. 1953, ApJ, 117, 313

Kelleher, D. E., \& Podobedova, L. I. 2008, J. Phys. Chem. Ref. Data, 37, No. 3, 1285

Kling, R., \& Griesmann, U. 2000, ApJ, 531, 1173

Kling, R., Schnabel, R., \& Griesmann, U. 2001, ApJS, 134, 173

Kocer, D., Adelman, S. J., Caliskan, H., Gulliver, A. F., \& Gokmen Tektunali, H. 2003, A\&A, 406, 975

Kochukhov, O. 2007, Spectrum synthesis for magnetic, chemically stratified stellar atmospheres, Physics of Magnetic Stars, 109, 118

Kochukhov, O., Tsymbal, V., Ryabchikova, T., Makaganyk, V., \& Bagnulo S. 2006, A\&A, 460, 831

Kontizas, E., \& Theodossiou, E. 1980, MNRAS, 192, 745

Kupka, F., Piskunov, N. E., Ryabchikova, T. A., Stempels, H. C., \& Weiss, W. W. 1999, A\&AS, 138, 119

Kurucz, R. L. 1993, SYNTHE spectrum synthesis programs and line data

Kurucz, R. L., \& Peytremann, E. 1975, SAO Special Report, 362

Kurucz, R. L. 1988, Trans. IAU, XXB, ed., M. McNally (Dordrecht: Kluwer), 168

Lacy, C. H. S., Fekel, F. C., Mathieu, R. D., et al. 1997, AJ, 113, 1088

Landstreet, J. D. 1998, A\&A, 338, 1041

Lanz, T., Dimitrijevic, M. C., \& Artru, M.-C. 1988, A\&A, 192,249

Lanz, T., Cunha, K., Holtzman J., \& Hubeny, I. 2008, ApJ, 678, 1342

Lawler, J. E., \& Dakin, J. T. 1989, JOSA B6, 1457

Lemke, M. 1990, A\&A, 225, 125

Leone, F., \& Lanzafame, A. C. 1998, A\&A, 330, 306

Lilly, R. A. 1976, JOSA, 66, 971

Ljung G., Nilsson H., Asplund M., \& Johansson S. 2006, A\&A, 456, 1181

Maíz-Apellániz, J. 2005, PASP, 117, 615

Maíz-Apellániz, J. 2006, AJ, 131, 1184

Malagnini, M. L., Faraggiana, R., \& Morossi, C. 1983, A\&A, 128, 375

Malfait, K., Bogaert, E., \& Waelkens, C. 1998, A\&A, 331, 211

Martin, G. A., Fuhr, J. R., \& Wiese, W. L. 1988, J. Phys. Chem. Ref. Data, 17, Suppl. 3

Mashonkina, L., Ryabchikova, T., \& Ryabtsev, A. 2005, A\&A, 441, 309

Matheron, P., Escarguel, A., Redon, R., Lesage, A., \& Richou, J. 2001, JQSRT, 69, 535

Megessier, C. 1988, A\&AS, 72, 551 
Miles, B. M., \& Wiese, W. L. 1969, WSG Technical Note, 474

Miller, M. H., Roig, R. A., \& Bengston, R. D. 1971, Phys. Rev. A, 4, 1709

Miller, M. H., Wilkerson, T. D., Roig, R. A., \& Bengston, R. D. 1974, Phys. Rev. A, 9,2312

Moon, T. T., \& Dworetsky, M. M. 1985, MNRAS, 217, 305

Morel, T., \& Butler, K. 2008, A\&A, 487, 307

Morossi, C., \& Malagnini, M. L. 1985, A\&AS, 60, 365

Napiwotzki, R., Schoenberner, D., \& Wenske, V. 1993, A\&A, 268, 653

Netopil, M., Paunzen, E., Maitzen, H. M., North, P., \& Hubrig, S. 2008, A\&A, 491, 545

Nicolet, B. 1978, A\&AS, 34,

Nieva, M. F., \& Przybilla, N. 2008, A\&A, 481, 199

Nilsson, H., Ljung, G., Lundberg, H., \& Nielsen, K. E. 2006, A\&A, 445, 1165

North, P., \& Nicolet, B. 1990, A\&A, 228, 78

O'Brian, T. R., \& Lawler, J. E. 1991, Phys. Rev. A, 44, 7134

O'Brian, T. R., Wickliffe, M. E., Lawler, J. E., Whaling, W., \& Brault, J. W. 1991, JOSA B8, 1185

Pamyatnykh, A. A. 1999, Acta Astronomica, 49, 119

Pickering, J. C., Thorne, A. P., \& Perez, R. 2001, ApJS, 132, 403

Piskunov, N. E., Kupka, F., Ryabchikova, T. A., Weiss, W. W., \& Jeffery, C. S. 1995, A\&AS, 112, 525

Przybilla, N., \& Butler, K. 2001, A\&A, 379, 955

Przybilla, N., Butler, K., Becker, S. R., Kudritzki, R. P., \& Venn, K. A. 2000, A\&A, 359, 1085

Przybilla, N., Butler, K., \& Kudritzki, R. P. 2001a, A\&A, 379, 936

Przybilla, N., Butler, K., Becker, S. R., \& Kudritzki, R. P. 2001b, A\&A, 369 1009

Przybilla, N., Nieva, M.-F., \& Butler, K. 2008, ApJ, 688, L103

Raassen, A. J. J., \& Uylings, P. H. M. 1998, A\&A, 340, 300

Raassen, A. J. J., Pickering, J. C., \& Uylings, P. H. M. 1998, A\&AS, 130, 541

Ralchenko, Yu., Kramida, A. E., Reader, J., \& NIST ASD Team, 2008, NIST Atomic Spectra Database, version 3.1.5, available: http: //physics .nist . gov/asd3, NIST, Gaithersburg, MD

Rentzsch-Holm, I. 1996, A\&A, 312, 966

Rentzsch-Holm, I. 1997, A\&A, 317, 178

Roby, S. W., \& Lambert, D. L. 1990, ApJS, 73, 67

Rufener, F. 1988, Catalogue of stars measured in the Geneva Observatory photometric system, 4, Sauverny: Observatoire de Geneve

Ryabchikova T. A., Hill, G. M., Landstreet J. D., Piskunov, N., \& Sigut, T. A. A 1994, MNRAS, 267, 697

Ryabchikova, T. A., Piskunov, N. E., Stempels, H. C., Kupka, F., \& Weiss, W. W. 1999, Phis. Scr., T83, 162

Ryabchikova, T. A., Wade, G. A., \& LeBlanc. F. 2003, in Modelling of Stellar Atmospheres, Proc. 210th IAU Symp., ed. N. Piskunov, W. W. Weiss, D. F. Gray, 301
Ryabchikova, T. Leone, F. \& Kochukhov, O. 2005, A\&A, 438, 973

Ryabchikova, T., Ryabtsev, A., Kochukhov, O., \& Bagnulo, S. 2006, A\&A, 456, 329

Sadakane, K. 1981, PASP, 93, 587

Sadakane, K., Jugaku, J., \& Takada-Hidai, M. 1988, ApJ, 325, 776

Schiller, F., \& Przybilla, N. 2008, A\&A, 479, 849

Schulz-Gulde, E. 1969, JQSRT, 9, 13

Schlegel, D. J., Finkbeiner, D. P., \& Davis, M. 1998, ApJ, 500, 525

Seaton, M. J., Mihalas, D., \& Pradhan, A. K. 1994, MNRAS, 266, 805

Shulyak, D., Tsymbal, V., Ryabchikova, T., Stütz, Ch., \& Weiss, W. W. 2004, A\&A, 428, 993

Sigut, T. A. A. 1999, ApJ, 519, 303.

Singh, J., \& Castelli, F. 1992, A\&A, 253, 431

Smith, G. 1988, J. Phys. B., 21, 2827

Smith, G., \& Gallagher, A. 1966, Phys. Rev., 145, 26

Smith, G., \& O'Neil, J. A. 1975, A\&A, 38, 1

Smith, G., \& Raggett, D. St. J. 1981, J. Phys. B, 14, 4015

Smith, K. C. 1993, A\&A, 276, 393

Smith, K. C. 1994, A\&A, 291, 521

Smith, K. C., \& Dworetsky, M. M. 1993, A\&A, 274, 335

Smith, M. W., \& Wiese, W. L. 1971, ApJS, 23, 103

Sobeck, J. S., Lawler, J. E., \& Sneden, C. 2007, ApJ, 667, 1267

Takeda, Y. 1994, PASJ, 46, 181

Takeda, Y. 2008, MNRAS, 388, 913

Theodosiou, E. 1989, Phys. Rev. A, 39, 4880

Theodossiou, E., \& Danezis, E. 1991, A\&AS, 183, 91

Tsymbal, V., Lyashko, D., \& Weiss, W. W. 2003, IAU Symp., 210, ed. N., Piskunov, W. W., Weiss, \& D. F., Gray, 49

Wade, G. A., Donati, J.-F., Landstreet, J. D., \& Shorlin, S. L. S. 2000, MNRAS, 313,823

Wade, G. A., Ryabchikova, T. A., Bagnulo, S., \& Piskunov, N. 2001, in Magnetic Fields Across the Hertzsprung-Russell Diagram, ed. G. Mathys, S. K. Solanki, \& D. T. Wickramasinghe, ASP Conf. Proc., 248, 373

Wahlgren, G. M., \& Hubrig, S. 2000, A\&A, 362, L13

Warner, B. 1968a, MNRAS, 139, 115

Warner, B. 1968b, MNRAS, 140, 53

Wedemeyer, S. 2001, A\&A, 373, 998

Wickliffe M. E., \& Lawler J. E. 1997a, ApJS, 110, 163

Wiese, W. L., \& Fuhr, J. R. 2007, J. Phys. Chem. Ref. Data, 36, 1287

Wiese, W. L., Smith, M. W., \& Glennon, B. M. 1966, NSRDS-WSG, 4

Wiese, W. L., Smith, M. W., \& Miles, B. M. 1969, NSRDS-WSG, 22

Wilke, R. 2003, Ph.D. Thesis, Heinrich-Heine-Universität, Düsseldorf

Yoon, J., Peterson, D. M., Zagarello, R. J., Armstrong, J. T., \& Pauls, T. 2008, ApJ, 681, 570 
L. Fossati et al.: The abundance analysis for normal A- and B-type stars, Online Material p 1

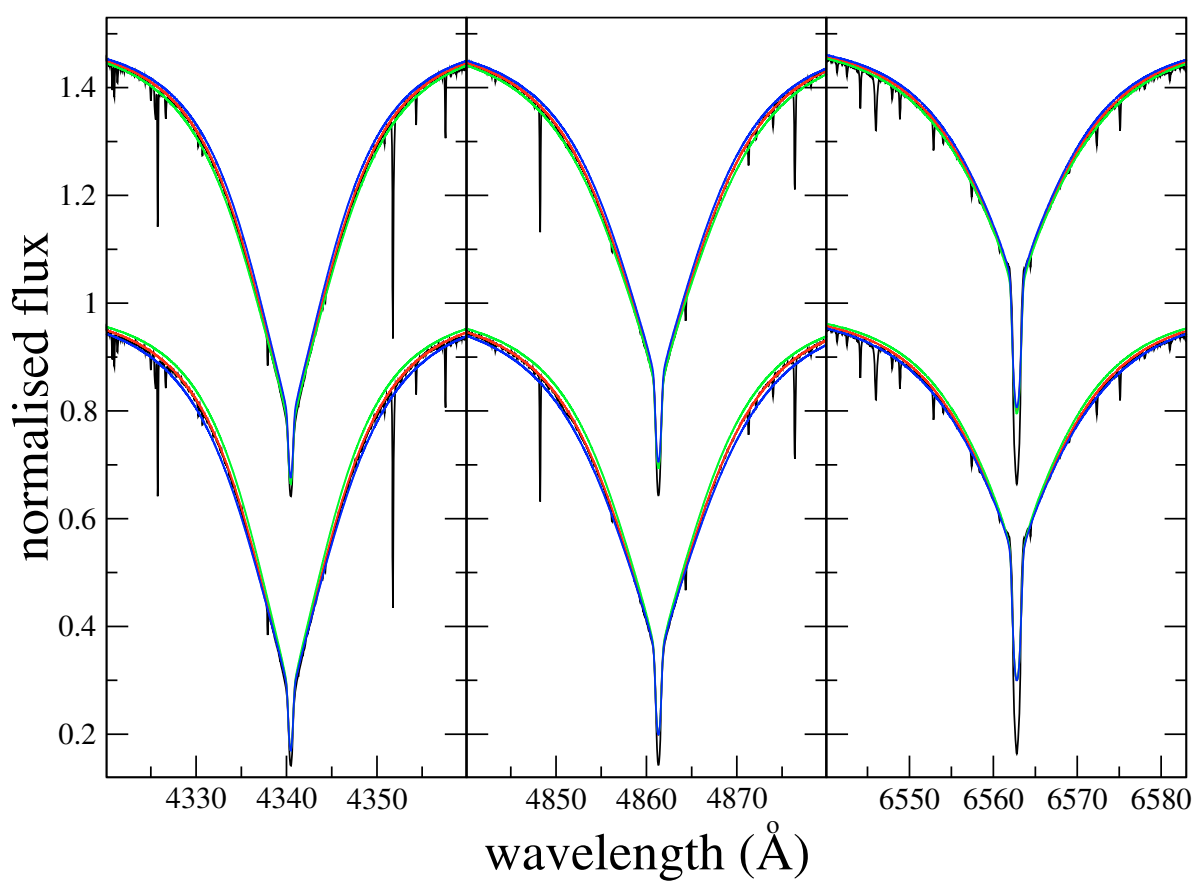

Fig. 8. Same as Fig. 2, but also for $\mathrm{H} \gamma$ and $\mathrm{H} \alpha$.

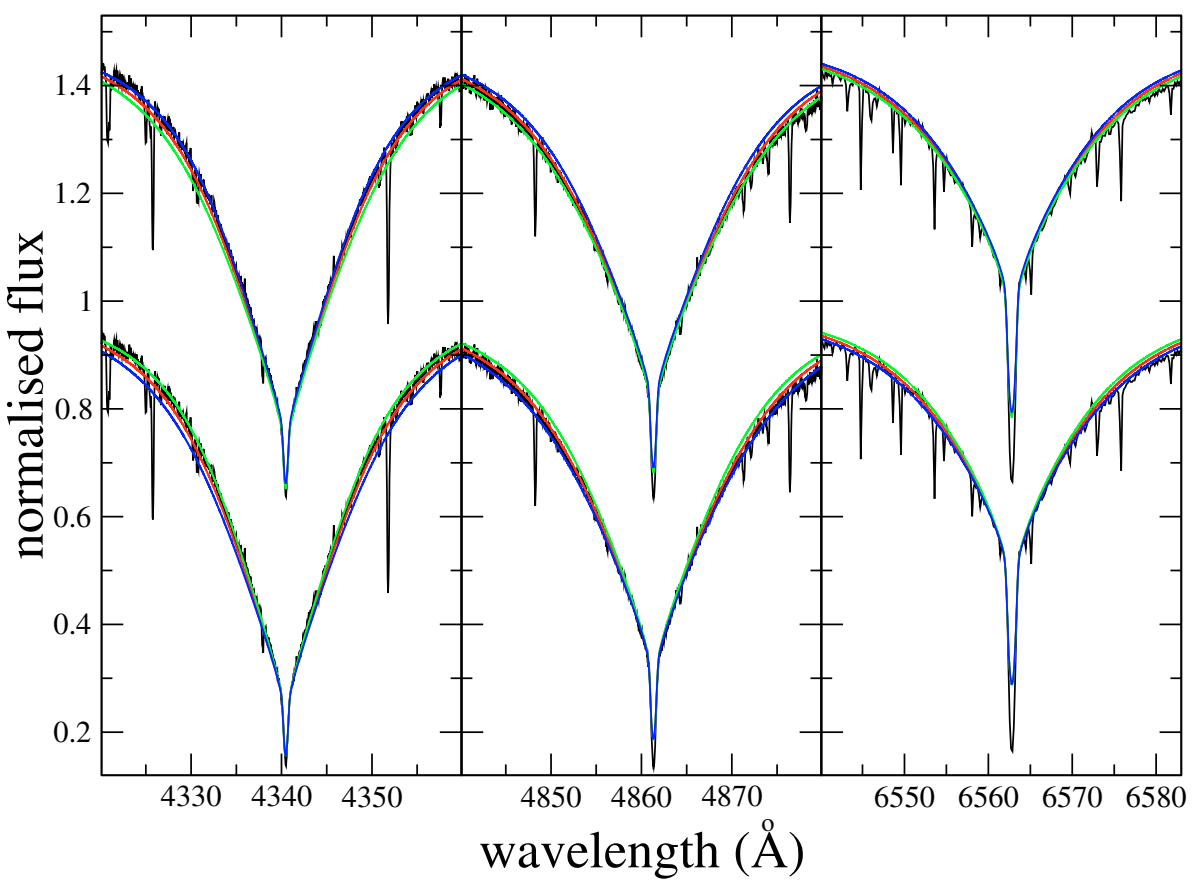

Fig. 9. Same as Fig. 8, but for HD 145788. 
L. Fossati et al.: The abundance analysis for normal A- and B-type stars, Online Material p 2

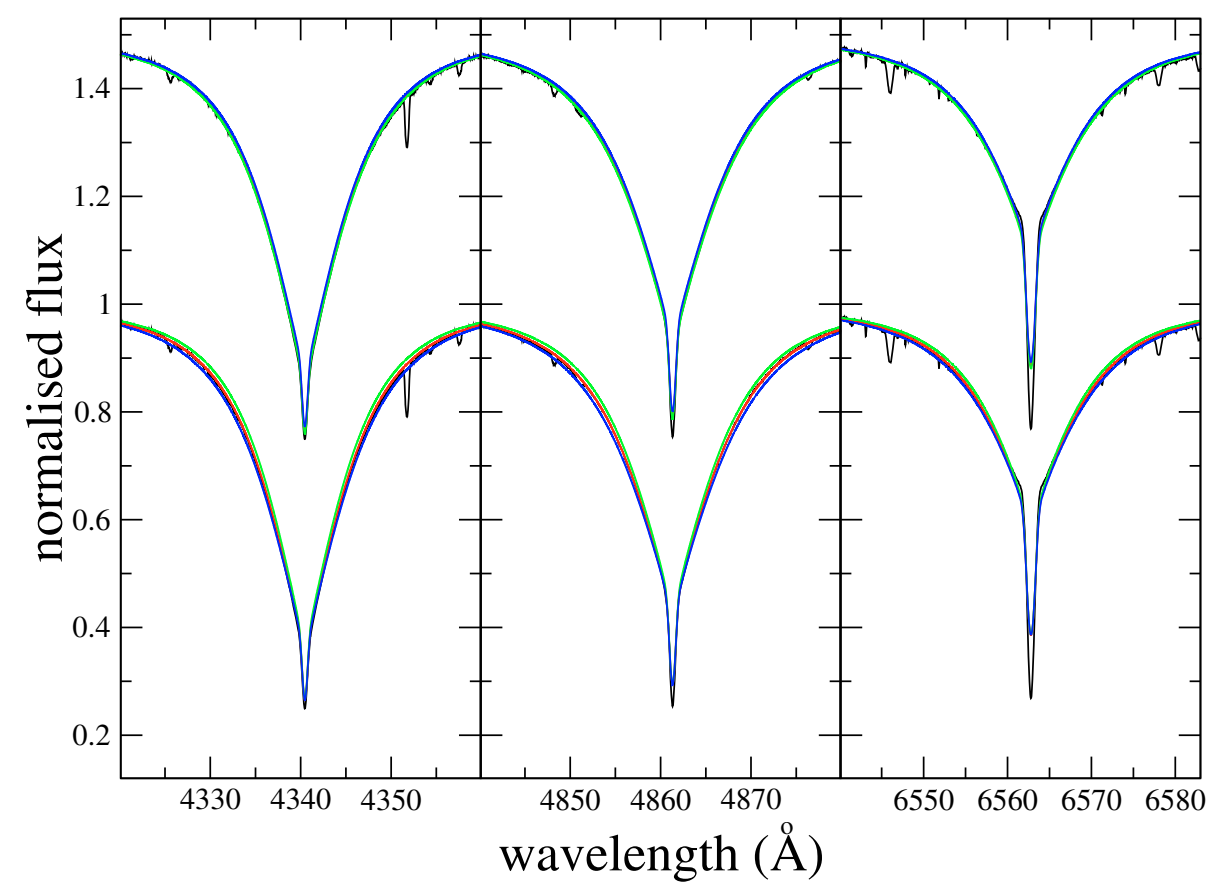

Fig. 10. Same as Fig. 8, but for $\pi$ Cet.

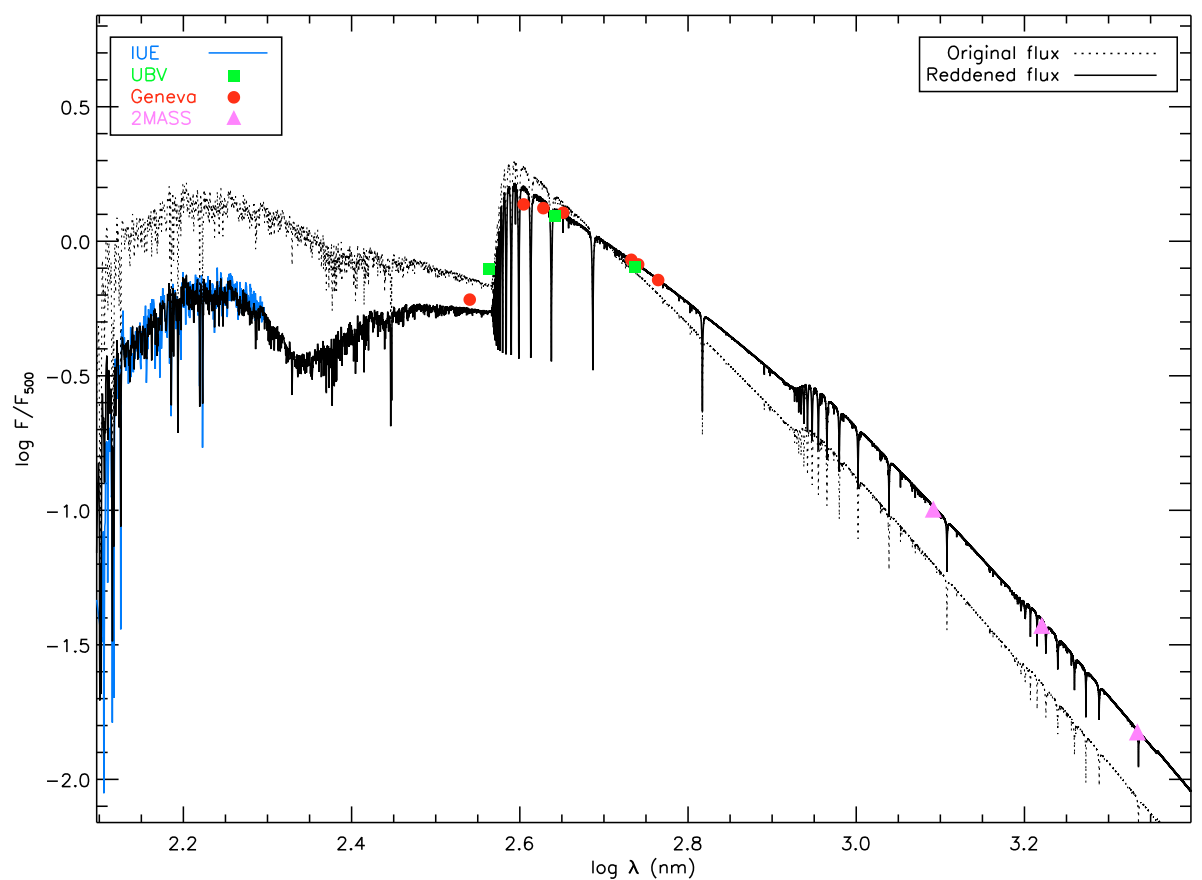

Fig. 11. Comparison between LLMODELS theoretical fluxes calculated with the fundamental parameters and abundances derived for HD 145788, taking into account a reddening of $E(B-V)=0.20$ (full black line) and without taking into account reddening (dashed black line), with IUE calibrated fluxes (full blue line), Johnson $U B V$ photometry (green squares), Geneva photometry (red circles) and 2MASS photmetry (violet triangles). The model fluxes were convolved to have approximately the same spectral resolution of the IUE fluxes $(R \sim 900)$. 
L. Fossati et al.: The abundance analysis for normal A- and B-type stars, Online Material p 3

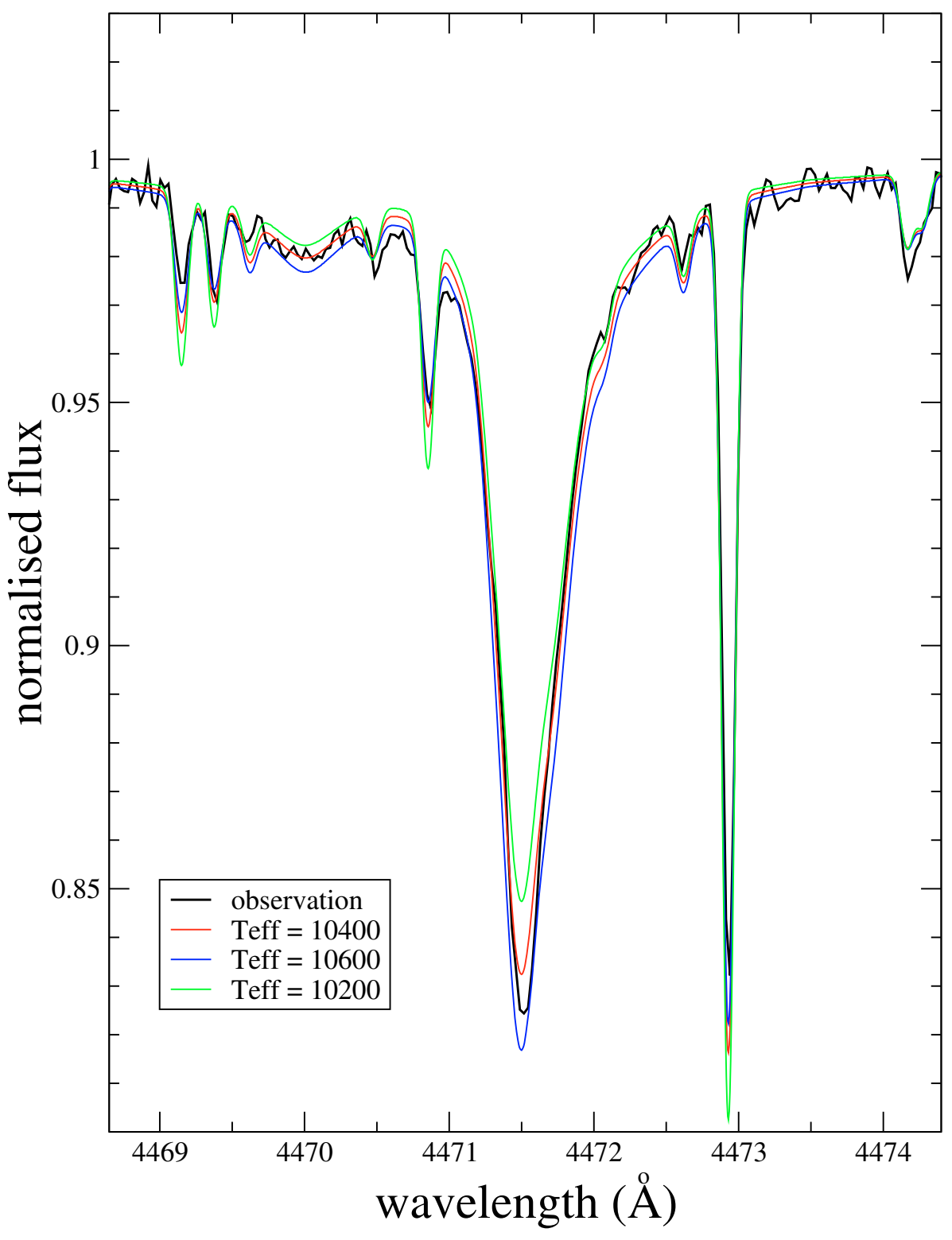

Fig. 12. Comparison between the observed spectrum of the He I line at $\lambda \sim 4471 \AA$ for 21 Peg (black thick line) and three synthetic profiles calculated with three different $T_{\text {eff }}$ for the model atmosphere: $10200 \mathrm{~K}$ (green line), the adopted $10400 \mathrm{~K}$ (red line) and $10600 \mathrm{~K}$ (blue line). 
L. Fossati et al.: The abundance analysis for normal A- and B-type stars, Online Material p 4

Table 6. Comparison of the derived abundances with previous determinations for $21 \mathrm{Peg}$.

\begin{tabular}{|c|c|c|c|c|c|c|c|c|}
\hline Ion & $\begin{array}{r}\mathrm{R} 08 \\
\log \left(N / N_{\text {tot }}\right) \\
\end{array}$ & $n$ & $\begin{array}{c}\mathrm{S} 81 \\
\log \left(N / N_{\text {tot }}\right)\end{array}$ & $n$ & $\begin{array}{r}\mathrm{S} 934 \\
\log \left(N / N_{\text {tot }}\right)\end{array}$ & $n$ & $\begin{array}{c}\mathrm{D} 00 \\
\log \left(N / N_{\text {tot }}\right)\end{array}$ & \\
\hline He I & $-1.11 \pm 0.04$ & 7 & & & & & & \\
\hline $\mathrm{CI}$ & $-3.66 \pm 0.14$ & 9 & -3.88 & 2 & & & & \\
\hline C II & $-3.65 \pm 0.05$ & 4 & & & & & & \\
\hline $\mathrm{N}$ I & $-3.95 \pm 0.12$ & 4 & & & & & & \\
\hline $\mathrm{N}_{\text {II }}$ & $-3.90:$ & 1 & & & & & & \\
\hline O I & $-3.28 \pm 0.11$ & 18 & & & & & & \\
\hline $\mathrm{Ne} \mathrm{I}$ & $-3.76 \pm 0.05$ & 7 & & & & & $-3.60 \pm 0.16$ & 3 \\
\hline & -3.89 (non-LTE) & 1 & & & & & -3.82 (non-LTE) & 1 \\
\hline $\mathrm{NaI}$ & -5.60 : & 1 & & & & & & \\
\hline Mg I & $-4.42 \pm 0.12$ & 5 & & & & & & \\
\hline Mg II & $-4.56 \pm 0.03$ & 7 & $-4.72 \pm 0.13$ & 7 & $-4.34 \pm 0.05$ & 4 & & \\
\hline Al I & -5.89 & 2 & -6.05 & 2 & & & & \\
\hline Al II & $-5.70 \pm 0.10$ & 4 & & & -5.84 & 1 & & \\
\hline Al III & & & & & $-5.89 \pm 0.21$ & 2 & & \\
\hline Si I & -4.95 : & 1 & & & & & & \\
\hline Si II & $-4.49 \pm 0.13$ & 22 & -4.85 & 2 & $-4.63 \pm 0.07$ & 2 & & \\
\hline Si III & -4.26 : & 2 & & & & & & \\
\hline PII & $-6.37 \pm 0.06$ & 3 & & & & & & \\
\hline S II & $-4.86 \pm 0.13$ & 26 & & & & & & \\
\hline $\mathrm{CaI}$ & $-5.84 \pm 0.11$ & 3 & -5.76 & 1 & & & & \\
\hline Ca II & $-5.98 \pm 0.08$ & 5 & -6.24 & 1 & & & & \\
\hline Sc II & $-9.37 \pm 0.10$ & 7 & -9.47 & 3 & & & & \\
\hline Ti II & $-7.23 \pm 0.09$ & 59 & $-7.15 \pm 0.16$ & 26 & & & & \\
\hline V II & $-7.98 \pm 0.06$ & 9 & $-7.78 \pm 0.16$ & 6 & & & & \\
\hline CrI & $-6.29 \pm 0.09$ & 5 & & & & & & \\
\hline CrII & $-6.20 \pm 0.10$ & 68 & $-6.39 \pm 0.25$ & 17 & $-6.30 \pm 0.01$ & 5 & & \\
\hline Mn I & $-6.54 \pm 0.21$ & 5 & -6.08 & 2 & & & & \\
\hline Mn II & $-6.51 \pm 0.17$ & 19 & & & $-6.39 \pm 0.10$ & 3 & & \\
\hline $\mathrm{Fe} I$ & $-4.52 \pm 0.13$ & 108 & $-4.80 \pm 0.21$ & 32 & & & & \\
\hline Fe II & $-4.50 \pm 0.12$ & 406 & $-4.79 \pm 0.18$ & 23 & $-4.59 \pm 0.05$ & 10 & & \\
\hline Fe III & $-4.60 \pm 0.06$ & 3 & & & & & & \\
\hline Co II & $-6.75 \pm 0.18$ & 3 & & & $-6.01 \pm 0.01$ & 3 & & \\
\hline $\mathrm{Ni}$ I & $-5.71 \pm 0.05$ & 10 & & & & & & \\
\hline Ni II & $-5.61 \pm 0.09$ & 23 & -6.13 & 5 & $-5.70 \pm 0.02$ & 4 & & \\
\hline $\mathrm{Zn} \mathrm{I}$ & $-6.96:$ & 1 & & & $-7.29 \pm 0.11$ & 3 & & \\
\hline Sr II & $-9.10:$ & 2 & -9.18 & 2 & & & & \\
\hline Y II & $-9.76 \pm 0.15$ & 4 & -9.27 & 1 & & & & \\
\hline Zr II & $-9.48 \pm 0.28$ & 4 & -9.09 & 1 & & & & \\
\hline Ba II & $-9.19 \pm 0.06$ & 3 & & & & & & \\
\hline Nd III & $-10.09 \pm 0.07$ & 3 & & & & & & \\
\hline
\end{tabular}

In Col. 2, the meaning of a colon is the same as in Table 4.

R08: this work; S81: Sadakane (1981); S934: Smith \& Dworetsky (1993); Smith (1993, 1994); D00: Dworetsky \& Budaj (2000). 
L. Fossati et al.: The abundance analysis for normal A- and B-type stars, Online Material p 5

Table 7. Comparison of the derived abundances with previous dterminations for $\pi$ Cet.

\begin{tabular}{|c|c|c|c|c|c|c|c|c|c|c|}
\hline Ion & \multicolumn{2}{|l|}{ R08 } & \multicolumn{2}{|l|}{ A91 } & \multicolumn{2}{|l|}{ S934 } & $\begin{array}{ll}\mathrm{D} 00 \\
\end{array}$ & $n$ & \multicolumn{2}{|l|}{$\mathrm{A} 04$} \\
\hline $\mathrm{He} \mathrm{I}$ & $-0.97 \pm 0.04$ & 6 & $-1.11 \pm 0.06$ & 6 & & & & & & \\
\hline C II & $-3.58 \pm 0.07$ & 7 & $-3.81 \pm 0.07$ & 4 & & & & & & \\
\hline N I & $-4.03 \pm 0.13$ & 10 & & & & & & & & \\
\hline N II & $-3.74 \pm 0.07$ & 9 & $-3.92 \pm 0.15$ & 3 & & & & & & \\
\hline O I & $-3.06 \pm 0.14$ & 9 & -3.34 & 2 & & & & & $-3.29 \pm 0.03$ & 4 \\
\hline O II & $-3.04:$ & 2 & & & & & & & & \\
\hline $\mathrm{Ne} I$ & $-3.66 \pm 0.09$ & 20 & & & & & $-3.71 \pm 0.19$ & 6 & & \\
\hline & $\begin{array}{l}-3.86 \text { (NLTE) } \\
-523+0.07\end{array}$ & 1 & & & & & -3.86 (NLTE) & 1 & & \\
\hline $\mathrm{Mg}_{\mathrm{I}}$ & $-4.27:$ & 2 & -4.88 & 1 & & & & & & \\
\hline $\mathrm{Mg}_{\text {II }}$ & $-4.47 \pm 0.16$ & 10 & $-4.56 \pm 0.08$ & 7 & & & & & & \\
\hline $\mathrm{Al} \mathrm{I}$ & $-5.57:$ & 2 & -5.85 & 2 & & & & & & \\
\hline $\mathrm{Al} \mathrm{II}$ & $-5.73 \pm 0.27$ & 8 & & & -6.04 & 1 & & & & \\
\hline $\mathrm{Al}$ III & $-5.30 \pm 0.02$ & 3 & -5.36 & 1 & $-5.79 \pm 0.10$ & 2 & & & & \\
\hline Si I & -4.80 & 1 & & & & & & & & \\
\hline Si II & $-4.41 \pm 0.20$ & 31 & $-4.56 \pm 0.12$ & 5 & $-4.44 \pm 0.05$ & 2 & & & $-4.44 \pm 0.28$ & 4 \\
\hline Si III & $-4.16:$ & 2 & -4.99 & 1 & & & & & & \\
\hline P II & $-6.38 \pm 0.19$ & 9 & & & & & & & & \\
\hline P III & $-6.19:$ & 1 & & & & & & & & \\
\hline S II & $-4.78 \pm 0.16$ & 31 & $-4.86 \pm 0.18$ & 18 & & & & & -4.90 & 1 \\
\hline $\mathrm{Cl}$ II & $-6.95:$ & 2 & & & & & & & & \\
\hline ArI & $-4.86:$ & 2 & & & & & & & & \\
\hline Ar II & $-5.24 \pm 0.19$ & 6 & & & & & & & & \\
\hline Ca II & $-5.77:$ & 2 & -5.72 & 1 & & & & & & \\
\hline Sc II & -9.31: & 1 & & & & & & & & \\
\hline Ti II & $-7.42 \pm 0.08$ & 11 & $-7.17 \pm 0.24$ & 14 & & & & & $-7.23 \pm 0.13$ & 7 \\
\hline Cr II & $-6.41 \pm 0.10$ & 21 & $-6.58 \pm 0.19$ & 15 & $-6.00 \pm 0.2$ & 5 & & & $-6.58 \pm 0.08$ & 10 \\
\hline Mn II & $-6.50 \pm 0.09$ & 3 & & & $-6.44 \pm 0.05$ & 3 & & & & \\
\hline $\mathrm{Fe} I$ & $-4.53 \pm 0.22$ & 7 & & & & & & & & \\
\hline Fe II & $-4.58 \pm 0.14$ & 186 & $-4.66 \pm 0.20$ & 59 & $-4.55 \pm 0.05$ & 10 & & & $-4.78 \pm 0.19$ & 29 \\
\hline Fe III & $-4.52 \pm 0.10$ & 4 & -4.82 & 1 & & & & & & \\
\hline Co II & $-6.93:$ & 1 & & & $-6.30 \pm 0.1$ & 3 & & & & \\
\hline Ni II & $-5.76 \pm 0.19$ & 17 & -6.02 & 3 & $-5.80 \pm 0.2$ & 4 & & & $-5.89 \pm 0.02$ & 2 \\
\hline Sr II & -9.15: & 2 & & & & & & & & \\
\hline & $v_{\text {mic }}=1 \mathrm{~km}$ & & $v_{\text {mic }}=0 \mathrm{kms}$ & & $v_{\text {mic }}=0 \mathrm{~km} \mathrm{~s}$ & & & & $v_{\text {mic }}=3 \mathrm{~km}$ & \\
\hline
\end{tabular}

In Col. 2, the meaning of a colon is the same as in Table 4.

R08: this work; A91: Adelman (1991); S934: Smith \& Dworetsky (1993); Smith (1993, 1994); D00: Dworetsky \& Budaj (2000); A04: Acke \& Waelkens (2004). 
L. Fossati et al.: The abundance analysis for normal A- and B-type stars, Online Material p 6

Table 8. A collection of the experimental and theoretical transition probabilities and Stark widths for the observed Si II lines. Errors are given in parenthesis.

\begin{tabular}{|c|c|c|c|c|c|c|c|c|c|c|c|}
\hline \multirow{2}{*}{$\begin{array}{l}\text { Wavelength } \\
\AA\end{array}$} & \multirow{2}{*}{$\begin{array}{r}\chi_{\text {excit }} \\
\text { eV }\end{array}$} & \multirow[b]{2}{*}{ NIST } & \multirow[b]{2}{*}{ Barach } & \multicolumn{4}{|c|}{$\overline{\overline{\log g f}}$} & \multirow[b]{2}{*}{ Wilke } & \multirow[b]{2}{*}{ AJPP } & \multicolumn{2}{|c|}{$\overline{\log \gamma_{\mathrm{St}}}$} \\
\hline & & & & SG & BBCB & $\mathrm{BBC}$ & Math & & & Wilke & LDA \\
\hline 3853.665 & 6.858 & -1.341 & & $-1.32(06)$ & -1.52 & $-1.39(06)$ & $-1.28(05)$ & $-1.53(11)$ & -1.44 & -5.12 & -5.15 \\
\hline 3856.018 & 6.859 & -0.406 & & $-0.34(06)$ & -0.56 & $-0.43(05)$ & $-0.36(06)$ & $-0.65(10)$ & -0.49 & -5.15 & -5.15 \\
\hline 3862.595 & 6.858 & -0.757 & & $-0.83(06)$ & -0.82 & $-0.80(06)$ & $-0.80(07)$ & $-0.92(10)$ & -0.75 & -5.14 & -5.15 \\
\hline 4072.709 & 9.837 & -2.700 & & $-2.37(05)$ & & & $-2.70(10)$ & $-2.40(16)$ & -1.89 & -4.89 & -4.76 \\
\hline 4075.452 & 9.839 & -1.403 & & $-1.40(05)$ & & & $-1.40(10)$ & $-1.64(16)$ & -0.95 & -4.89 & -4.76 \\
\hline 4076.780 & 9.837 & -1.700 & & $-1.67(05)$ & & & $-1.70(10)$ & $-1.75(16)$ & -1.20 & -4.89 & -4.76 \\
\hline 4128.054 & 9.837 & 0.359 & & $0.45(04)$ & 0.32 & & $0.36(12)$ & $0.22(08)$ & 0.38 & -4.92 & -4.88 \\
\hline 4130.872 & 9.839 & -0.783 & & & -0.82 & & & & -0.77 & & -4.88 \\
\hline 4130.894 & 9.839 & 0.552 & & $0.50(04)$ & 0.48 & & $0.55(12)$ & $0.38(08)$ & 0.53 & -4.93 & -4.88 \\
\hline 4190.707 & 13.492 & & & & & & $-0.17(10)$ & $-0.33(11)$ & -0.35 & -5.25 & -5.26 \\
\hline 4198.134 & 13.487 & & & & & & $-1.46(11)$ & $-0.60(11)$ & -0.61 & -5.26 & -5.26 \\
\hline 4200.658 & 12.525 & -0.889 & & & & & & & & & -3.43 \\
\hline 4621.418 & 12.525 & -0.608 & & & & & & & & & -3.69 \\
\hline 4621.722 & 12.526 & -0.453 & & & & & & & & & -3.69 \\
\hline 5041.023 & 10.067 & 0.029 & & $0.29(03)$ & & $0.09(04)$ & $0.03(07)$ & $0.18(03)$ & 0.19 & -4.84 & -4.78 \\
\hline 5055.983 & 10.074 & 0.523 & & $0.59(03)$ & & $0.46(04)$ & $0.52(08)$ & $0.48(03)$ & 0.44 & -4.79 & -4.78 \\
\hline 5056.316 & 10.074 & -0.492 & & $-0.36(03)$ & & $-0.49(04)$ & & & -0.51 & -4.79 & -4.78 \\
\hline 5466.460 & 12.525 & -0.237 & & & & & & & & & -3.99 \\
\hline 5469.451 & 12.880 & -0.762 & & & & & & & & & \\
\hline 5540.807 & 14.489 & & $-0.83(08)$ & & & & $-1.20(10)$ & & -0.80 & & \\
\hline 5576.661 & 14.504 & & $-0.47(08)$ & & & & $-0.47(10)$ & $-0.62(13)$ & -0.49 & -5.46 & \\
\hline 5632.966 & 14.186 & -0.818 & $-0.62(08)$ & & & $-0.82(06)$ & & $-0.82(09)$ & -0.69 & -5.44 & \\
\hline 5639.477 & 14.528 & & $-0.07(06)$ & & & $-0.30(07)$ & $-0.07(12)$ & $-0.18(09)$ & -0.32 & -5.44 & \\
\hline 5669.563 & 14.200 & 0.286 & $0.32(06)$ & & & $0.28(07)$ & & $0.12(08)$ & 0.25 & -5.53 & \\
\hline 5688.817 & 14.186 & 0.126 & $0.08(06)$ & & & $0.13(06)$ & & $0.00(09)$ & 0.08 & -5.50 & \\
\hline 5701.370 & 14.175 & -0.057 & $-0.10(06)$ & & & $-0.06(07)$ & & $-0.28(09)$ & -0.10 & -5.53 & \\
\hline 5800.454 & 14.504 & & $-0.04(08)$ & & & $-0.16(07)$ & $-0.12(12)$ & $-0.16(11)$ & -0.17 & -5.37 & \\
\hline 5806.731 & 14.489 & & $-0.10(08)$ & & & $-0.13(07)$ & $-0.11(12)$ & $-0.18(10)$ & -0.20 & -5.47 & \\
\hline 5827.752 & 14.489 & & $-0.91(18)$ & & & $-0.79(07)$ & $-1.00(10)$ & $-1.16(11)$ & -0.90 & -5.45 & \\
\hline 5846.121 & 14.504 & & $-0.51(08)$ & & & $-0.29(07)$ & $-0.57(12)$ & $-0.33(14)$ & -0.70 & -5.35 & \\
\hline 5867.480 & 14.504 & & & & & & & & -0.20 & & \\
\hline 5868.443 & 14.528 & & $0.42(06)$ & & & & $0.40(14)$ & $0.50(11)$ & 0.20 & -5.36 & \\
\hline 5957.559 & 10.067 & -0.225 & & $-0.30(02)$ & & $-0.22(03)$ & & $-0.29(06)$ & -0.33 & -5.02 & -4.84 \\
\hline 5978.930 & 10.074 & 0.084 & & $0.00(02)$ & & $0.08(03)$ & & $0.03(06)$ & -0.03 & -5.01 & -4.84 \\
\hline 6347.109 & 8.121 & 0.149 & & $0.18(05)$ & 0.30 & $0.20(04)$ & $0.12(07)$ & $0.29(08)$ & 0.17 & -5.31 & -5.05 \\
\hline 6371.371 & 8.121 & -0.082 & & $-0.06(05)$ & -0.00 & $-0.03(03)$ & $-0.04(07)$ & $-0.02(08)$ & -0.13 & -5.32 & -5.05 \\
\hline 6660.532 & 14.504 & 0.162 & & & & & & $0.23(09)$ & 0.24 & -5.51 & \\
\hline 6665.030 & 14.495 & -0.240 & & & & & & $-0.18(09)$ & -0.16 & -5.54 & \\
\hline 6671.841 & 14.528 & 0.409 & & & & $0.41(07)$ & & $0.46(09)$ & 0.52 & -5.58 & \\
\hline 6699.431 & 14.495 & -0.247 & & & & $-0.25(08)$ & & & -0.16 & & \\
\hline 7848.816 & 12.525 & 0.316 & & & & & & & 0.34 & & -4.34 \\
\hline 7849.618 & 12.526 & -0.831 & & & & & & & -0.80 & & -4.34 \\
\hline 7849.722 & 12.526 & 0.470 & & & & & & & 0.50 & & -4.34 \\
\hline
\end{tabular}

NIST - Kelleher \& Podobedova (2008) - critical compilation; Barach - Barach (1970) - pulsed arc; SG - Schilz-Gulde (1969) - arc emission spectra; BBCB - Berry et al. (1971) - beam-foil lifetime measurements and theortical branching ratios; BBC - Blanco et al. (1995) - laser-induced plasma; Math - Matheron et al. (2001) - laser-induced plasma; Wilke - Wilke (2003) - laser-induced plasma, Stark widths, shifts and transition probabilities; AJPP - Artru et al. (1981) - theoretical calculations; LDA - Lanz et al. (1988) - Stark widths, semi-classical calculations. 